\title{
Precision test of the muon-Higgs coupling at a high-energy muon collider
}

Tao Han, ${ }^{a}$ Wolfgang Kilian, ${ }^{b}$ Nils Kreher, ${ }^{b}$ Yang Ma, ${ }^{a}$ Jürgen Reuter, ${ }^{c}$ Tobias Striegl ${ }^{b}$ and Keping $\mathrm{Xie}^{a}$

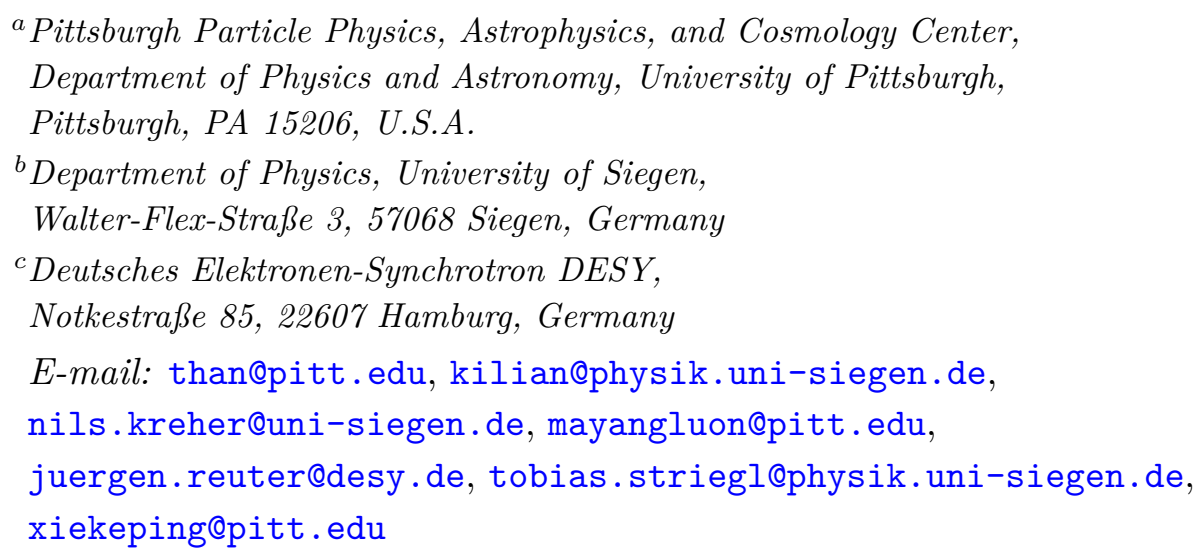

ABSTRACT: We explore the sensitivity of directly testing the muon-Higgs coupling at a high-energy muon collider. This is strongly motivated if there exists new physics that is not aligned with the Standard Model Yukawa interactions which are responsible for the fermion mass generation. We illustrate a few such examples for physics beyond the Standard Model. With the accidentally small value of the muon Yukawa coupling and its subtle role in the high-energy production of multiple (vector and Higgs) bosons, we show that it is possible to measure the muon-Higgs coupling to an accuracy of ten percent for a $10 \mathrm{TeV}$ muon collider and a few percent for a $30 \mathrm{TeV}$ machine by utilizing the three boson production, potentially sensitive to a new physics scale about $\Lambda \sim 30-100 \mathrm{TeV}$.

Keywords: Beyond Standard Model, Effective Field Theories, Higgs Physics

ArXiv EPrint: 2108.05362 


\section{Contents}

1 Introduction 1

2 Theoretical considerations for the muon Yukawa coupling 3

2.1 Illustrations of the running of the muon Yukawa coupling 3

$\begin{array}{lll}2.2 & \text { EFT description of an anomalous muon Yukawa coupling } & 7\end{array}$

2.2.1 The Yukawa interaction in the HEFT parameterization $\quad 7$

2.2.2 The Yukawa interaction in the SMEFT parameterization 9

2.2.3 Unitarity bounds on a nonstandard Yukawa sector 12

$\begin{array}{lll}2.2 .4 & \text { Multi-boson production and cross section ratios } & 14\end{array}$

3 Phenomenology of muon-Higgs coupling at a high-energy muon collider 20

$\begin{array}{lll}3.1 & \text { Multi-boson production } & 20\end{array}$

$\begin{array}{lll}3.2 & \text { Kinematic distributions } & 24\end{array}$

$\begin{array}{lll}3.3 & \text { Statistical sensitivity on the muon Yukawa coupling } 26\end{array}$

$\begin{array}{llr}4 & \text { Summary and conclusions } & 29\end{array}$

\section{Introduction}

The Standard Model (SM) of elementary particle physics is constructed based on a nonAbelian gauge theory of $\mathrm{SU}(3)_{\mathrm{C}} \otimes \mathrm{SU}(2)_{\mathrm{L}} \otimes \mathrm{U}(1)_{\mathrm{Y}}$, that has been experimentally verified with a high accuracy to the highest energies accessible to date [1]. On the other hand, there is mounting evidence from observations for the need of new physics beyond the SM, such as the dark matter, neutrino mass generation, and the matter/antimatter asymmetry.

Unlike the past decades, at the moment we are lacking well-defined traces of where to look for new physics. While there are many loose ends in the SM of particle physics and cosmology, however, there is no clear indication at what energy scales new phenomena would appear below the Planck scale. This gives us the task to use all available tools to search for new phenomena, particularly all the discovered particles as vehicles for our searches. Especially, the scalar boson discovered in $2012[2,3]$ which closely resembles the SM Higgs boson is very well suited for beyond the Standard Model (BSM) searches [4]. Currently, the couplings of the Higgs boson to the third generation SM fermions have been established with a precision of $10 \%-20 \%$ (for an overview of the current status and projections, see e.g. [5]). The high-luminosity phase of the LHC will study the properties of this particle and its couplings to a precision at a few percent level [6, 7]. The next collider facility will most likely be a Higgs factory $[8,9]$ in the form of an electron-positron collider running at or slightly above the $Z H$ threshold, such as the International Linear Collider (ILC) $[10,11]$, the Future Circular Collider (FCC-ee) [12], the Circular Electron-Positron 
Collider (CEPC) [13], or the Compact Linear Collider (CLIC) at higher energies [14, 15] to achieve a per-mille level accuracy for the Higgs couplings to $W^{+} W^{-}, Z Z, \gamma \gamma, g g$ and $b \bar{b}, \tau \bar{\tau}, c \bar{c}$, as well as the invisible decay mode.

However, there will still be parts of the Higgs sector left unexplored or measured with low precision because it can only be probed with very rare processes for which there are too low rates at a Higgs factory and the LHC measurements (or searches) suffer from large systematic uncertainties due to the challenging experimental environment. To this class belong the couplings to the first and second generations of fermions. The Higgs mechanism in the SM provides the mass for all elementary particles, and thus specifies the form of their interactions associated with the electroweak symmetry breaking (EWSB). With only a single $\mathrm{SU}(2)_{L}$ Higgs doublet and the minimal set of interactions at the renormalizable level, the Yukawa couplings of SM fermions are proportional to the respective particle masses, and thus exhibit a large hierarchy. It would be desirable to achieve a better precision for the measurement of the Yukawa couplings of the light fermions, since this would be a direct and important test whether the Higgs mechanism as implemented in the SM provides the masses for all SM fermions, or whether it is a mixture of two (or more) mechanisms. Because of the small Yukawa couplings for light fermions predicted in the SM, any small deviation due to BSM physics may result in a relatively large modification to those couplings.

The next target is the Higgs-muon coupling. The recent evidence for the $H \rightarrow \mu^{+} \mu^{-}$ decay at ATLAS and CMS indicates that the Yukawa coupling is present within the predicted order of magnitude $[16,17]$. However, the results are not yet at the $5 \sigma$ level for discovery, and thus leaves room for $O(100 \%)$ corrections. Also, the measurement is insensitive to the sign of the coupling. According to the current experimental projections, by the end of the high-luminosity runs of the LHC in the late 2030s the muon Yukawa coupling could be measured with an accuracy of about several tens of percent [18] in a model-dependent way. This situation might not be improved very much neither at the Higgs factory due to the limited rate, nor at a high-energy hadron collider like the FCChh $[19,20]$, due to the systematics and the model-dependence. Thanks to the technological development [21], a renewed idea that has recently gathered much momentum is the option of a high-energy muon collider that could reach the multi-(tens of) $\mathrm{TeV}$ regime with very high luminosity [22-24]. It has been demonstrated in the recent literature that a highenergy muon collider has great potential for new physics searches at the energy frontier from direct $\mu^{+} \mu^{-}$annihilation and a broad reach for new physics from the rich partonic channels [25-29], as well as precision measurements for SM physics [30] and beyond [31-39]. Of particular importance is the connection between the muon collider expectation and the tantalizing hint for new physics from the muon $g-2$ measurement [40, 41].

In this paper, we propose one unique measurement and BSM search in the Higgs sector which serves as a paradigm example for exploiting a high-energy muon collider, namely the direct measurement of the muon Yukawa coupling. At a high-energy $\mu^{+} \mu^{-}$collider, one probes the coupling at a much higher energy scale and it may reach some sensitivity to new physics with scale-dependent effects. Unlike the precision measurements at low energies where one probes the virtual quantum effects, our proposal is to directly measure 
the muon coupling associated with its mass generation. Our search strategy is generally applicable to other new physics searches involving final states of charged leptons and jets, that may provide general guidance for future considerations.

The rest of the paper is organized as follows. We first present a brief overview and motivation for the importance of studies of the muon Yukawa coupling in section 2. In section 2.1, we examine the renormalization group (RG)-induced scale dependence of the couplings. This is important to relate a measured quantity in a high-energy collider setup to the low-scale value. In section 2.2, we construct an effective field theory (EFT) setting to discuss possible deviations of the muon Yukawa coupling from its SM value. We present a few paradigm examples of modifications of the muon-Higgs coupling from its SM Yukawa value. In section 2.2.2 we then discuss different EFT parameterizations, constraints from unitarity limits in section 2.2.3, and consequences for ratios of different production cross sections in section 2.2.4. It sets the theoretical frame for our phenomenological studies in section 3, where we analyze the collider sensitivity for the determination of the muon Yukawa coupling at a high energy muon collider, before we conclude in section 4 .

\section{Theoretical considerations for the muon Yukawa coupling}

\subsection{Illustrations of the running of the muon Yukawa coupling}

When testing the muon-Higgs Yukawa coupling, it is necessary to properly take into account the energy-scale dependence of the coupling, which is a fundamental prediction in quantum field theory. The specific form of this running depends on the particle spectrum and their interactions in the underlying theory. In the electroweak sector of the SM, the dominant contribution to the renormalization group (RG) running is the top Yukawa coupling, followed by the strong and EW gauge interactions.

For the sake of illustration, the coupled renormalization group equations (RGEs) of Yukawa couplings $y_{\mu}, y_{t}$, vacuum expectation value $v$, and gauge couplings $g_{i}$ are given in the $\overline{\mathrm{MS}}$ scheme at leading order (LO) in one-loop by [42-48]

$$
\begin{aligned}
& \beta_{y_{t}}=\frac{\mathrm{d} y_{t}}{\mathrm{~d} t}=\frac{y_{t}}{16 \pi^{2}}\left(\frac{9}{2} y_{t}^{2}-8 g_{3}^{2}-\frac{9}{4} g_{2}^{2}-\frac{17}{20} g_{1}^{2}\right), \\
& \beta_{y_{\mu}}=\frac{\mathrm{d} y_{\mu}}{\mathrm{d} t}=\frac{y_{\mu}}{16 \pi^{2}}\left(3 y_{t}^{2}-\frac{9}{4}\left(g_{2}^{2}+g_{1}^{2}\right)\right), \\
& \beta_{v}=\frac{\mathrm{d} v}{\mathrm{~d} t}=\frac{v}{16 \pi^{2}}\left(\frac{9}{4} g_{2}^{2}+\frac{9}{20} g_{1}^{2}-3 y_{t}^{2}\right), \\
& \beta_{g_{i}}=\frac{\mathrm{d} g_{i}}{\mathrm{~d} t}=\frac{b_{i} g_{i}^{3}}{16 \pi^{2}},
\end{aligned}
$$

with $t=\ln \left(Q / M_{Z}\right)$ and the coefficients $b_{i}$ for the gauge couplings $\left(g_{1}, g_{2}, g_{3}\right)$ given as

$$
b_{i}^{\mathrm{SM}}=(41 / 10,-19 / 6,-7) .
$$

We show the LO RGE running of the muon Yukawa $y_{\mu}$ in the SM in figure 1 (red solid curve) and the SM vacuum expectation value $v$ in figure 2 (left axis) as functions of the 


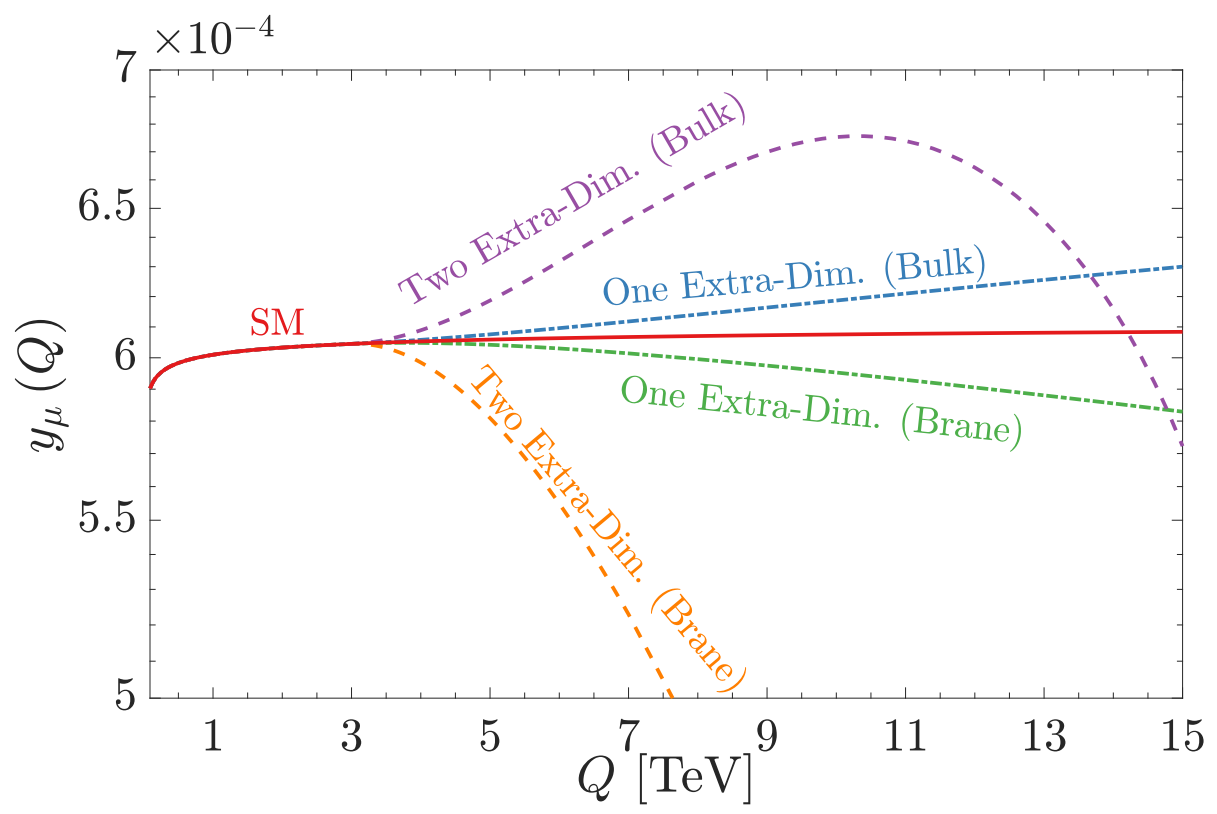

Figure 1. LO RGE running of the muon Yukawa $y_{\mu}$ coupling as a function of the energy scale $Q$, in the $\mathrm{SM}$ (red solid). In the extra-dimensional scenarios (with inverse radius $1 / R=3 \mathrm{TeV}$ ), we consider 1) Bulk: all fields propagating in the bulk, and 2) Brane: all matter fields localized to the brane.

energy scale $Q$, respectively. With the relation

$$
m_{\mu}(Q)=y_{\mu}(Q) v(Q) / \sqrt{2}
$$

we also show the running of the muon mass, $m_{\mu}(Q)$, in figure 2 (right axis). At the energy scales accessible in near future colliders, the change in $y_{\mu}$ is observed to be rather small, for example, $y_{\mu}(Q=15 \mathrm{TeV})$ is found to be around $3 \%$ smaller compared to $y_{\mu}\left(M_{Z}\right)$. Similarly, $v\left(m_{\mu}\right)$ runs down by about $4 \%(2 \%)$.

New states appearing in beyond SM scenarios can modify the running of the relevant gauge and Yukawa couplings. Generically, the beta function for a coupling $\lambda$ is given as

$$
\beta_{\lambda}=\beta_{\lambda}^{\mathrm{SM}}+\sum_{\text {s: massive new states }} \theta\left(Q-M_{s}\right) \times N_{s} \beta_{s, \lambda}^{\mathrm{NP}},
$$

where $\beta_{\lambda}^{\mathrm{SM}}$ is the SM beta function, and $\beta_{s, \lambda}^{\mathrm{NP}}$ represents the contribution of a new heavy state $s$ of mass $M_{s}$, with $N_{s}$ number of degenerate degrees of freedom. The theta function encodes the fact that the effect of new heavy states is included in the RG running once the energy scale $Q$ is above the threshold $M_{s}$, ignoring here for simplicity the effect of threshold corrections.

In extensions of the SM, the muon-Higgs Yukawa coupling could also be affected both at the tree level and at the quantum level. In addition, the Higgs sector may show a rich flavor structure. In flavor-sensitive Higgs models, the SM prediction for the Yukawa couplings is lost, and the Yukawa couplings become free model parameters. The physical coupling of the SM Higgs to muons may be larger or smaller than its expected SM value. 


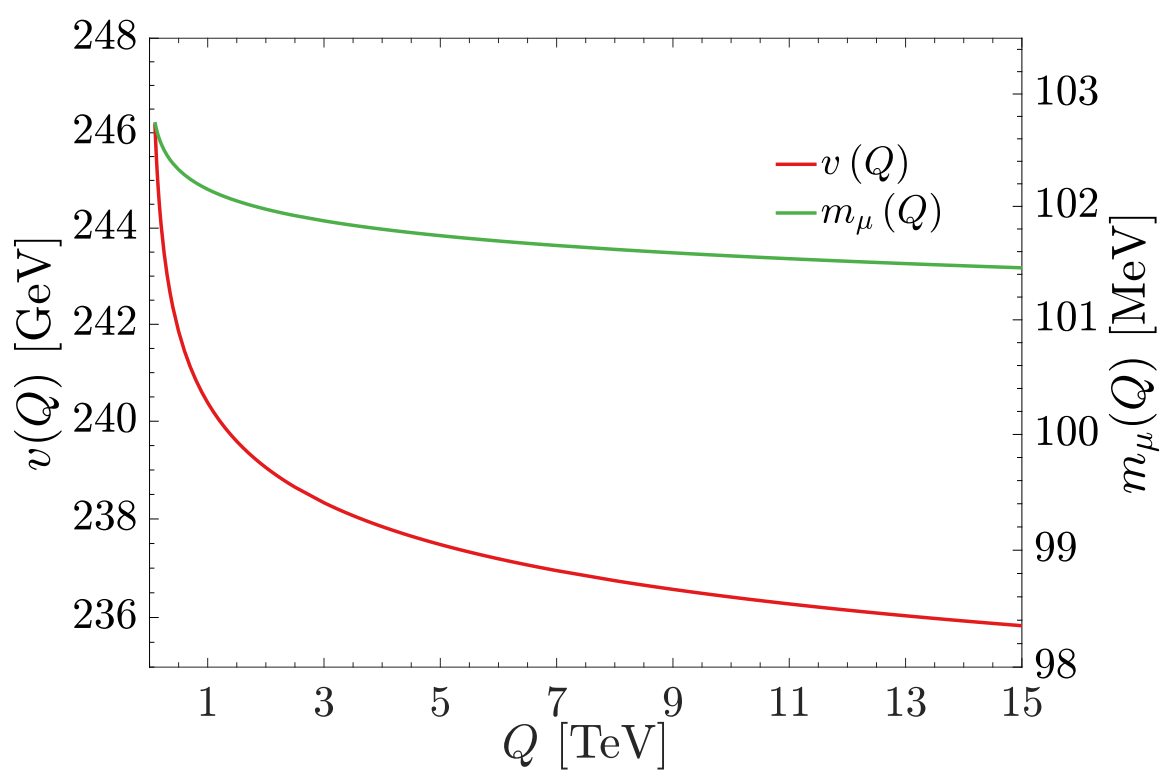

Figure 2. LO RGE running of SM vacuum expectation value $v$ (left scale) and muon mass $m_{\mu}$ (right scale) as functions of the energy scale $Q$.

In principle, it could be completely absent, such that the muon mass is generated by other means. The assumption we make for the study in this paper is that the muon Yukawa coupling is a free parameter, as the mass generation for the muon is in general a mixture of the SM mechanism and a yet-unknown mechanism. A typical example for this is a Two-Higgs doublet model $(2 \mathrm{HDM})$, or in a general multi-doublet model, that generates third-generation Yukawa couplings, while the second generation couplings are from a different sector (a sample implementation of such a mechanism can be found in [49]). Clearly, the LHC offers also some opportunities to probe first and second generation Higgs Yukawa couplings to light quarks [50], which applies mostly to the Higgs charm Yukawa coupling [51-54], and maybe even strange tagging is possible at a future Higgs factory [55]. In weakly-coupled theories, the running effects for the muon-Yukawa coupling are rather moderate, similar in size to that in the SM. We will not show it separately.

An interesting question is also whether there could be considerable $\mathrm{CP}$ violation in the Higgs Yukawa sector beyond CKM, where there are bounds e.g. for the electron Yukawa coupling [56]. Though it is perfectly possible in our setup in section 2.2 to discuss CPviolating operators for the muon Yukawa couplings, such a study is beyond the scope of this current paper.

We add the remark that additional, flavor-dependent, higher-dimensional operators that are responsible for a deviation of the SM muon Yukawa coupling could easily lead to flavor-violating Yukawa couplings that induced $H \rightarrow e \mu$. This has been studied e.g. in [57], however, we are not further investigating such flavor-violating processes in this paper. The EFT setup for our study is presented in detail in the next section.

Large modifications to the running couplings compared to the SM case are not expected in four-dimensional quantum field theories essentially due to the logarithmic nature of the 
running. A qualitatively different scenario however is obtained if there is a tower of new physics states modifying the RGEs, asymptotically leading to a power-law running of the Yukawa coupling $[58,59]$. This four-dimensional description is equivalent to a theory with compactified flat extra space-like dimensions, with gauge and/or matter fields propagating in the higher-dimensional bulk. To illustrate this, we consider two scenarios of compactified flat extra-dimensions [60]: a 5D model with the extra-dimension compactified on an $S_{1} / Z_{2}$ orbifold, and a $6 \mathrm{D}$ model with the two extra dimensions compactified on a square $T^{2} / Z_{2}$ orbifold [60,61]. In both models, we consider two cases: 1) all SM fields propagating in the bulk and 2) the SM gauge fields to be propagating in the bulk, with the matter fields of the SM restricted to the brane [62-66]. The beta functions of the gauge couplings in such scenarios are given as:

$$
\begin{aligned}
& b_{i}^{5 \mathrm{D}}=b_{i}^{\mathrm{SM}}+(S(t)-1) \times\left[\left(\frac{1}{10},-\frac{41}{6},-\frac{21}{2}\right)+\frac{8}{3} \eta\right] \\
& b_{i}^{6 \mathrm{D}}=b_{i}^{\mathrm{SM}}+\left(\pi S(t)^{2}-1\right) \times\left[\left(\frac{1}{10},-\frac{13}{2},-10\right)+\frac{8}{3} \eta\right] .
\end{aligned}
$$

Here, $S(t)$ counts the number of degrees of freedom $S(t)=e^{t} R, R$ being the radius of the extra dimension, $\eta$ being the number of generations of fermions propagating in the bulk. The corresponding one-loop RGE equations for the Yukawa couplings $y_{t}, y_{\mu}$ in the extra-dimensional scenarios are as follows $[63,66,67]$

$$
\begin{array}{rlrl}
\frac{d y_{t}}{d t} & =\beta_{y_{t}}^{\mathrm{SM}}+\frac{y_{t}}{16 \pi^{2}} 2(S(t)-1)\left(\frac{3}{2} y_{t}^{2}-8 g_{3}^{2}-\frac{9}{4} g_{2}^{2}-\frac{17}{20} g_{1}^{2}\right), & & \text { 5D Brane, } \\
\frac{d y_{\mu}}{d t} & =\beta_{y_{\mu}}^{\mathrm{SM}}-\frac{y_{\mu}}{16 \pi^{2}} 2(S(t)-1)\left(\frac{9}{4} g_{2}^{2}+\frac{9}{4} g_{1}^{2}\right), & & \text { 5D Brane, } \\
\frac{d y_{t}}{d t} & =\beta_{y_{t}}^{\mathrm{SM}}+\frac{y_{t}}{16 \pi^{2}}(S(t)-1)\left(\frac{15}{2} y_{t}^{2}-\frac{28}{3} g_{3}^{2}-\frac{15}{8} g_{2}^{2}-\frac{101}{120} g_{1}^{2}\right), & & \text { 5D Bulk, } \\
\frac{d y_{\mu}}{d t} & =\beta_{y_{\mu}}^{\mathrm{SM}}+\frac{y_{\mu}}{16 \pi^{2}}(S(t)-1)\left(6 y_{t}^{2}-\frac{15}{8} g_{2}^{2}-\frac{99}{40} g_{1}^{2}\right), & & \text { 5D Bulk. } \\
\frac{d y_{t}}{d t}=\beta_{y_{t}}^{\mathrm{SM}}+\frac{y_{t}}{16 \pi^{2}} 4 \pi\left(S(t)^{2}-1\right)\left(\frac{3}{2} y_{t}^{2}-8 g_{3}^{2}-\frac{9}{4} g_{2}^{2}-\frac{17}{20} g_{1}^{2}\right), & & \text { 6D Brane, } \\
\frac{d y_{\mu}}{d t}=\beta_{y_{\mu}}^{\mathrm{SM}}-\frac{y_{\mu}}{16 \pi^{2}} 4 \pi\left(S(t)^{2}-1\right)\left(\frac{9}{4} g_{2}^{2}+\frac{9}{4} g_{1}^{2}\right), & & \text { 6D Brane, } \\
\frac{d y_{t}}{d t}=\beta_{y_{t}}^{\mathrm{SM}}+\frac{y_{t}}{16 \pi^{2}} \pi\left(S(t)^{2}-1\right)\left(9 y_{t}^{2}-\frac{32}{3} g_{3}^{2}-\frac{3}{2} g_{2}^{2}-\frac{5}{6} g_{1}^{2}\right), & & \text { 6D Bulk, } \\
\frac{d y_{\mu}}{d t}=\beta_{y_{\mu}}^{\mathrm{SM}}+\frac{y_{\mu}}{16 \pi^{2}} \pi\left(S(t)^{2}-1\right)\left(6 y_{t}^{2}-\frac{3}{2} g_{2}^{2}-\frac{27}{10} g_{1}^{2}\right), & & \text { 6D Bulk. }
\end{array}
$$

We see from figure 1 that in the presence of such a tower of new states, the running of $y_{\mu}$ can be substantially altered for both the 5D (dot-dashed curves), and 6D (dashed curves) models. We note that the effects only become significant when close or above the new physics threshold, $1 / R \sim 3 \mathrm{TeV}$ in our illustration. Above the threshold, the other more direct effects from the existence of the extra dimensions may be observable as well and a coordinated search would be beneficial. 
We conclude that while in the SM the energy dependence of the $y_{\mu}$ is a minor effect, there are viable models where the value and the running of this quantity could both follow completely different patterns, as illustrated above with extra-dimensional scenarios. In the next subsection, we will extend this direction in the EFT framework.

\subsection{EFT description of an anomalous muon Yukawa coupling}

In a purely phenomenological ansatz, if small modifications of the SM Lagrangian exist, they should be detectable most easily in interactions which are accidentally suppressed in the SM, and at the same time are unaffected by large radiative corrections. The muon mass and the associated production and decay processes perfectly fit this scenario. In this spirit, we introduce representative new interactions in form of a modification of this muon mass parameter, without referencing a specific model context. The modification is supposed to be tiny in absolute terms, but nevertheless becomes significant if compared with the SM muon Yukawa coupling which has a numerical value of less than $10^{-3}$. A few well-motivated physics scenarios with a modification of the SM can be constructed as we will discuss next. They may describe rather different underlying dynamics, but represent physically equivalent calculational frameworks in the perturbative regime.

\subsubsection{The Yukawa interaction in the HEFT parameterization}

In the Higgs Effective Theory (HEFT) [68-73] or non-linear chiral-Lagrangian description, the scalar sector consists of a physical singlet Higgs boson together with unphysical triplet Goldstone bosons associated with the EW symmetry breaking. The latter isolate the contributions of longitudinally polarized vector bosons. This property can be formalized as the Goldstone-boson Equivalence Theorem (GBET) [74, 75]:

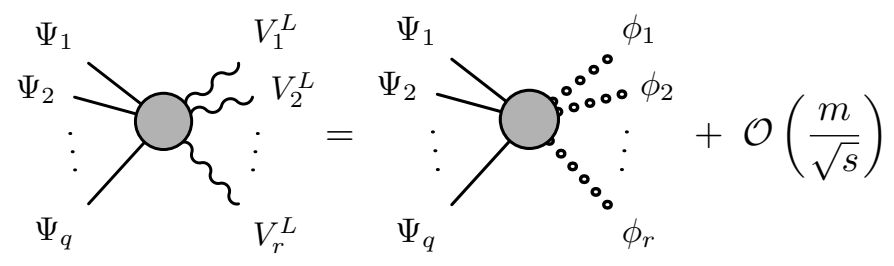

Here, $V_{k}^{L}$ denotes a longitudinal EW vector boson, $\phi_{k}$ the corresponding Goldstone boson, and $\Psi_{k}$ any possible SM fermion. This denotes that fact that matrix elements for multiboson final states including vector bosons are dominated in the high-energy limit by their longitudinal component

$$
\varepsilon_{L}^{\mu}(p)=\frac{p^{\mu}}{m}+v_{p}^{\mu}
$$

where $v_{p}^{\mu} \sim \mathcal{O}(m / \sqrt{s})$ is a four-vector depending on the boson momentum. According to [76] the GBET in an EFT framework takes the form

$$
\begin{aligned}
\mathcal{M}\left(V_{1}^{L}, \ldots, V_{r}^{L}, \boldsymbol{\Phi}\right)= & \left(\prod_{j}^{r} \pm i \omega_{j}\right) \mathcal{M}^{0}\left(\phi_{1}, \ldots, \phi_{r}, \mathbf{\Phi}\right) \\
& +\mathcal{O}\left(\frac{m}{\sqrt{s}}\right)+\mathcal{O}\left(\frac{\sqrt{s}}{\Lambda}\right)^{N+1}+\mathcal{O}\left(g, g^{\prime}\right),
\end{aligned}
$$


where $\mathcal{M}^{0}$ is the leading order of the matrix element in $g, g^{\prime}$, and $\mathcal{O}\left(g, g^{\prime}\right)$ denotes terms, which are suppressed by $g, g^{\prime}$ in comparison to this leading term. The $\omega_{j}$ are specific phases that differ between initial and final states within the amplitude. In this framework, the matrix elements appear not only as series expansions in the gauge couplings, but also in $\sqrt{s} / \Lambda$, which are usually truncated after some finite order $N$. The high-energy scale $\Lambda$ of any such bottom-up EFT corresponds to a specific scale of BSM models, e.g. a reference mass of a single heavy new particle. All longitudinal gauge bosons $V_{i}^{L}$ can be replaced by the corresponding Goldstone bosons $\phi_{i}$ at high energies within the accuracy goal of the EFT. The results will match at the leading order in $g$ and $g^{\prime}$.

In the present context, we can rewrite a modified muon Yukawa coupling as a gaugeinvariant operator in the HEFT Lagrangian, and conclude that this new interaction should cause extra contributions to the production of multiple vector bosons in association with the Higgs boson which rise with energy. By construction, these contributions exactly reproduce the effect of spoiled gauge cancellations in unitary gauge, as computed by automated programs.

In the non-linear representation we introduce a field $U$

$$
U=e^{i \phi^{a} \tau_{a} / v} \quad \text { with } \quad \phi^{a} \tau_{a}=\sqrt{2}\left(\begin{array}{cc}
\frac{\phi^{0}}{\sqrt{2}} & \phi^{+} \\
\phi^{-} & -\frac{\phi^{0}}{\sqrt{2}}
\end{array}\right),
$$

and its covariant derivative

$$
D_{\mu} U=\partial_{\mu} U+i g W_{\mu} U-i \frac{g^{\prime}}{2} B_{\mu} U \tau_{3} \quad \text { with } \quad W_{\mu}=\frac{1}{2} \tau_{a} W_{\mu}^{a},
$$

where $\tau_{a}$ denote the usual Pauli matrices and $\left\{\phi^{+}, \phi^{-}, \phi^{0}\right\}$ are the Goldstone bosons to the corresponding gauge bosons $\left\{W^{+}, W^{-}, Z\right\}$. The most general extension of the SM Lagrangian can be written as

$$
\mathcal{L}_{\mathrm{EW}}=-\frac{1}{2} \operatorname{tr} W_{\mu \nu} W^{\mu \nu}-\frac{1}{4} B_{\mu \nu} B^{\mu \nu}+\sum_{f \in\left\{\ell_{L}, \ell_{R}\right\}} i \bar{f}^{i} \not D f^{i}+\mathcal{L}_{U H}+\mathcal{L}_{\text {gauge-fix }} .
$$

The Higgs and Goldstone sector is given by

$$
\begin{aligned}
\mathcal{L}_{U H}= & \frac{v^{2}}{4} \operatorname{tr}\left[D_{\mu} U^{\dagger} D^{\mu} U\right] F_{U}(H)+\frac{1}{2} \partial_{\mu} H \partial^{\mu} H-V(H) \\
& -\frac{v}{2 \sqrt{2}}\left[\bar{\ell}_{L}^{i} \tilde{Y}_{\ell}^{i j}(H) U\left(1-\tau_{3}\right) \ell_{R}^{j}+\text { h.c. }\right]
\end{aligned}
$$

where we defined the right-handed doublets as $\ell_{R}^{i}=\left(\nu_{R}^{i}, e_{R}^{i}\right)^{T}$, and $i, j$ are the lepton-flavor indices. In the SM, the functions $F_{U}(H), V(H)$ and $Y_{e}^{i j}(H)$ are simple polynomials in $H / v$ that can be generalized to

$$
\begin{aligned}
F_{U}(H) & =1+\sum_{n \geq 1} f_{U, n}\left(\frac{H}{v}\right)^{n}, \\
V(H) & =v^{4} \sum_{n \geq 2} f_{V, n}\left(\frac{H}{v}\right)^{n} \quad \text { and } \\
\tilde{Y}_{\ell}^{i j}(H) & =\sum_{n \geq 0} \tilde{Y}_{\ell, n}^{i j}\left(\frac{H}{v}\right)^{n} .
\end{aligned}
$$


We do not assume CP violation in this sector, hence the coefficient of these different series are real, $\tilde{f}_{U, n}, f_{V, n}, \tilde{Y}_{\ell, n}^{i j} \in \mathbb{R}$. They are general parameters that can be obtained by a matching procedure from a possible underlying physical model, and in principle can be measured in appropriate physical processes.

We are primarily interested in the Higgs-lepton couplings. So we read off the mass matrix for the leptons

$$
\tilde{M}_{\ell}^{i j}=\frac{v}{\sqrt{2}} \tilde{Y}_{\ell, 0}^{i j}
$$

which is non-diagonal in general. As its eigenvalues are assumed to be positive, we can perform the usual polar decomposition $\tilde{M}_{\ell}=U_{L} M_{\ell} U_{R}^{\dagger}$ with some unitary matrices $U_{L / R}$ and compensate this by the rotation to the physical fields $\ell_{L} \mapsto U_{L} \ell_{L}$ and $\ell_{R} \mapsto U_{R} \ell_{R}$. Furthermore this defines $Y_{\ell, n}=U_{L}^{\dagger} \tilde{Y}_{\ell, n} U_{R}$, where, again, $n+1$ is the number of Higgs fields involved in the corresponding vertex. We will focus on the physical basis from now on. Note, that these equations all are still matrix equations, with the $(2,2)$-components $Y_{\ell, 0}^{2,2}:=y_{\mu}, Y_{\ell, n}^{2,2}:=y_{n}$ and $M_{\ell}^{2,2}:=m_{\mu}$ denoting the muon. Selecting the muon term and requiring the physical muon mass to equal its observed value, we observe an effective correction of the observable Yukawa coupling by the factor

$$
\kappa_{\mu}=\frac{v}{\sqrt{2} m_{\mu}} y_{1}
$$

which, for $y_{1}=y_{0}=y_{\mu}$, would correspond to the SM case $\kappa_{\mu}=1$. A priori, the size of the coupling coefficients is unknown as it depends on the underlying dynamics. From the "naive dimensional analysis" [77, 78], one would expect the modification as $y_{n} \sim y_{\mu}\left(g^{2} / 16 \pi^{2}\right)^{n}$, with $g \sim 1$ for a weakly coupled theory and $g \sim \mathcal{O}(4 \pi)$ a strongly coupled theory.

New operators in the series expansion in $H / v$ introduce contact terms which couple the muon to $n$ Higgs or Goldstone bosons. These contact terms are proportional to $y_{m}$, where $m \leq n$ denotes the number of Higgs bosons and they are the leading contributions to $\mu^{+} \mu^{-} \rightarrow n \varphi$ scattering in the high energy limit. Hence, via the GBET, a modification of $y_{\mu}$ is generically accompanied by new large contributions to multi-boson production in the high-energy limit.

\subsubsection{The Yukawa interaction in the SMEFT parameterization}

In the SMEFT framework, the SM gauge invariance is represented in linear form, and the Higgs boson combines with the Goldstone bosons as a complex SU(2) doublet. The pure effect of a modified muon Yukawa coupling can be reproduced by an infinite series of higher-dimensional operators in the SMEFT Lagrangian [79-82], where all coefficients are related to the original coupling modification. The results will be again identical to the unitary-gauge calculation.

However, if we furthermore assume a decoupling property of the new interactions, i.e., their parameters are not intrinsically tied to the electroweak scale, we should expect higherorder terms in the SMEFT series to be suppressed by a new heavy physics scale $v^{2} / \Lambda^{2}$, such that truncation after the first term is permissible. In that case, we have to discard the former relation between all orders, and accept that the resulting amplitudes will differ 
from the unitary-gauge results for an anomalous Yukawa coupling. In concrete terms, in a decoupling new-physics scenario we expect anomalous production of multiple vector bosons to be accompanied by anomalous production of multiple Higgs bosons. The clean environment of a muon collider is optimally suited to separate such final states irrespective of their decay modes, and thus to guide model building in either direction, depending on the pattern actually observed in data. The formalism set up here is very similar to the one used in [83] for searching deviations in the charm and strange Yukawa couplings in multi-boson production at the LHC and FCC-hh.

In the linear representation of the Higgs doublet,

$$
\varphi=\frac{1}{\sqrt{2}}\left(\begin{array}{c}
\sqrt{2} \phi^{+} \\
v+H+i \phi^{0}
\end{array}\right)
$$

the most general bottom-up extension of the SM Lagrangian,

$$
\begin{aligned}
\mathcal{L}_{\mathrm{EW}}= & -\frac{1}{2} \operatorname{tr} W_{\mu \nu} W^{\mu \nu}-\frac{1}{4} B_{\mu \nu} B^{\mu \nu}+\left(D_{\mu} \varphi\right)^{\dagger}\left(D^{\mu} \varphi\right)+\mu^{2} \varphi^{\dagger} \varphi-\frac{\lambda}{2}\left(\varphi^{\dagger} \varphi\right)^{2} \\
& +\sum_{f \in\left\{\ell_{L}, e_{R}\right\}} i \bar{f}^{i} \not D f^{i}-\left(\bar{\ell}_{L}^{i} \tilde{Y}_{\ell}^{i j} \varphi e_{R}^{j}+\text { h.c. }\right)+\mathcal{L}_{\text {gauge-fix }}
\end{aligned}
$$

that leads to a modification of the Yukawa coupling, reads

$$
\mathcal{L}=\mathcal{L}_{\mathrm{EW}}+\left[\sum_{n=1}^{N} \frac{\tilde{C}_{\ell \varphi}^{(n) i j}}{\Lambda^{2 n}}\left(\varphi^{\dagger} \varphi\right)^{n} \bar{\ell}_{L}^{i} \varphi e_{R}^{j}+\text { h.c. }\right] .
$$

Operators of higher mass dimension are as usual suppressed by a large scale $\Lambda$ that can be understood as an energy cutoff for the validity of the theory, as it will lead to an expansion of the scattering matrix elements in $\sqrt{s} / \Lambda$. Again, we do not consider CP violation, hence the Wilson coefficients are real $\tilde{C}_{\ell \varphi}^{(n)} \in \mathbb{R}$. They can be obtained by a matching procedure from an underlying physical model, and in principle can be measured. ${ }^{1}$ For further calculations, we absorb the large scale $1 / \Lambda^{2}$ in the Wilson coefficients.

We can read off the (non-diagonal) mass matrix for the charged leptons

$$
\tilde{M}_{\ell}^{i j}=\frac{v}{\sqrt{2}}\left(\tilde{Y}_{\ell}^{i j}-\sum_{n=1}^{N} \tilde{C}_{\ell \varphi}^{(n) i j} \frac{v^{2 n}}{2^{n}}\right) .
$$

In the same way as for the non-linear representation, we can diagonalize the mass matrix by redefinitions of the physical fields $e_{L} \mapsto U_{L} e_{L}, e_{R} \mapsto U_{R} e_{R}$. This defines $Y_{\ell}=U_{L}^{\dagger} \tilde{Y}_{\ell} U_{R}$ and $C_{\ell \varphi}^{(n)}=U_{L}^{\dagger} \tilde{C}_{\ell \varphi}^{(n)} U_{R}$.

As already discussed for the non-linear case, the operator coefficients $C_{\ell \varphi}^{(n)}$ can shift the muon Yukawa coupling away from its SM value. Because of its intrinsically small value, a moderate new physics contribution could lead to a drastic effect, driving it to zero or reversing its sign. The extreme case of a vanishing muon Yukawa coupling has the significant consequence that multi-Higgs production, $\mu^{+} \mu^{-} \rightarrow H^{M}$ would be absent

\footnotetext{
${ }^{1}$ One rather measures form factors, which are linear combinations of the Wilson coefficients.
} 
at tree level, while production of up to $k \in\{1, \ldots, M-1\}$ Higgs bosons associated with $M-k$ vector bosons would be allowed. As a paradigm example, we show how to embed this in our SMEFT framework: we require all lepton couplings to $k$ Higgs bosons, $\Lambda_{(k)}$, $k \in\{1, \ldots, M-1\}$, to vanish while the mass of the measured muon mass $m_{\mu}$ is fixed as an input. This leads to the conditions

$$
\begin{aligned}
M_{\ell} & =\frac{v}{\sqrt{2}}\left[Y_{\ell}-\sum_{n=1}^{M-1} C_{\ell \varphi}^{(n)} \frac{v^{2 n}}{2^{n}}\right] \\
\Lambda_{(k)} & :=-i \frac{k !}{\sqrt{2}}\left[Y_{\ell} \delta_{k, 1}-\sum_{n=n_{k}}^{M-1} C_{\ell \varphi}^{(n)}\left(\begin{array}{c}
2 n+1 \\
k
\end{array}\right) \frac{v^{2 n+1-k}}{2^{n}}\right]=0,
\end{aligned}
$$

where $n_{k}=\max \left(1,\left\lceil\frac{k-1}{2}\right\rceil\right)$.

For the general case, we define the following modification of the SM Yukawa coupling, still matrix-valued in flavor space, as

$$
K_{\ell}=1-\frac{v}{\sqrt{2}} M_{\ell}^{-1} \sum_{n=1}^{M-1} C_{\ell \varphi}^{(n)} \frac{n v^{2 n}}{2^{n-1}} .
$$

Again, we can project to the muon via $Y_{\ell}^{2,2}:=y_{\mu}, C_{\ell \varphi}^{(n) 2,2}:=c_{\ell \varphi}^{(n)}, M_{\ell}^{2,2}:=m_{\mu}$, as well as $K_{\ell}^{2,2}:=\kappa_{\mu}$.

As usual, we will consider the linear SMEFT expansion up to the first non-trivial order, which adds to the dimension-4 SM Yukawa coupling operator, $\mathcal{L}_{\text {Yuk. }}=-\left(\bar{\ell}_{L} Y_{\ell} e_{R}\right) \varphi$ at dimension- 6 a single operator that modifies the static Higgs coupling to leptons:

$$
\mathcal{O}_{\ell \varphi}=C_{\ell \varphi}\left(\varphi^{\dagger} \varphi\right)\left(\bar{\ell}_{L} e_{R}\right) \varphi
$$

Here, both $\Gamma_{\ell}$ as well as $C_{\ell \varphi}$ are matrices in lepton-flavor space. On dimensional grounds, $C_{\ell \varphi} \sim 1 / \Lambda^{2}$, where $\Lambda$ is the scale at which new physics sets in. Inserting the Higgs vev, we obtain at dimension- 4 the SM value of the lepton mass matrix, $M_{\ell}^{(4)}=\frac{v}{\sqrt{2}} Y_{\ell}$, while at dimension- 6 we get a modified mass matrix

$$
M_{\ell}^{(6)}=\frac{v}{\sqrt{2}}\left(Y_{\ell}-\frac{v^{2}}{2} C_{\ell \varphi}\right) .
$$

Specializing to the muon term and requiring the physical muon mass to equal its measured value, we observe an effective modification of the observable Yukawa coupling by the factor

$$
\kappa_{\mu}^{(6)}=1-\frac{v^{3}}{\sqrt{2} m_{\mu}} c_{\ell \varphi}^{(1)} .
$$

Expanding the Higgs field, the new operator induces contact terms which couple the muon to $n=1,2$, or 3 Higgs or Goldstone bosons. The contact terms are all proportional to the operator coefficient $c_{\ell \varphi}^{(1)}$, either scalar or pseudoscalar. Squaring this interaction, we obtain local contributions to $\mu^{+} \mu^{-} \rightarrow n \varphi$ scattering, in analogy with the HEFT description. The physical final states are Higgs or longitudinal $W, Z$ gauge bosons. As we will discuss in 
more detail in section 2.2.4, the $d=6$ contributions to their production cross sections with multiplicity $n=3$ rise with energy, $\sigma \propto s$, while the SM contribution falls off like $1 / s$. There is no interference, since — for these final states - the SM requires a vector exchange while the new contact term is scalar. We obtain a deviation from the SM prediction which is determined by the EFT contribution alone, which becomes leading above some threshold which depends on $\kappa_{\mu}^{(6)}-1$. The decomposition of the anomalous contribution into particle types ( $W W Z, W W h$, etc.) is fixed by electroweak symmetry and the particular SMEFT operator content, such that the exclusive channels are related by simple rational factors beyond the threshold where the new-physics part starts to dominate the production rates. This will be elaborated in section 2.2.4.

If the correction was large enough to render $\kappa_{\mu}=0$, we would obtain the unitarity bound for $d=6$, i.e. three-boson emission, as discussed in the next subsection. Generally speaking, the modification from the SM Yukawa coupling could reach an order of $100 \%$ if $c_{\ell \varphi}^{(1)} \sim 0.1 /(10 v)^{2}$. We emphasize that these two sample scenarios - a pure modified Yukawa coupling, and a modified Yukawa coupling combined with truncation of the SMEFT series - are to be understood as mere representatives of a potential new class of SM modifications that are difficult to observe at lower energy. As our results indicate, there is a great redundancy in the analysis of exclusive multi-boson final states, which should translate into significant discrimination power regarding more detailed models of the Higgs-Yukawa sector beyond the SM. If we translate an experimental bound on $\Delta \kappa_{\mu}$ to the SMEFT coefficient $c^{(1)} \sim g / \Lambda^{2}$, we obtain a bound on the scale of new physics as

$$
\Lambda>10 \mathrm{TeV} \sqrt{\frac{g}{\Delta \kappa_{\mu}}} .
$$

\subsubsection{Unitarity bounds on a nonstandard Yukawa sector}

In the SM, the high-energy asymptotics of the multi-boson production cross sections universally fall off with rising energy, manifesting themselves in delicate gauge cancellations which become huge at high energies. A modification of the muon Yukawa coupling from the SM prediction would show up as spoiling such cancellations, and thus eventually causes specific scattering amplitudes to rise again, without limits. While in theory, such a unitary-gauge framework does not do justice to the built-in symmetries of the SM, it is nevertheless the baseline framework for any tree-level evaluations such as the ones that we use in this work.

In ref. [84], generic models have been investigated where the leading contribution to a fermion mass originates from a dimension- $d$ EFT operator that couples the fermion to the SM Higgs field. Using the GBET, they computed the energy scale $\Lambda_{d}$ where unitarity is violated by multiple emission of Goldstone bosons, representing longitudinally polarized weak vector bosons, and Higgses.

$$
\Lambda_{d}=4 \pi \kappa_{d}\left(\frac{v^{d-3}}{m_{f}}\right)^{1 /(d-4)}, \quad \text { where } \quad \kappa_{d}=\left(\frac{(d-5) !}{2^{d-5}(d-3)}\right)^{1 /(2(d-4))} .
$$

For any given $d>4$, the most relevant bound corresponds to a final state that consists of $n=d-3$ Goldstone or Higgs bosons in total. For $m_{f}=m_{\mu}$ and $d=6,8,10$, the 


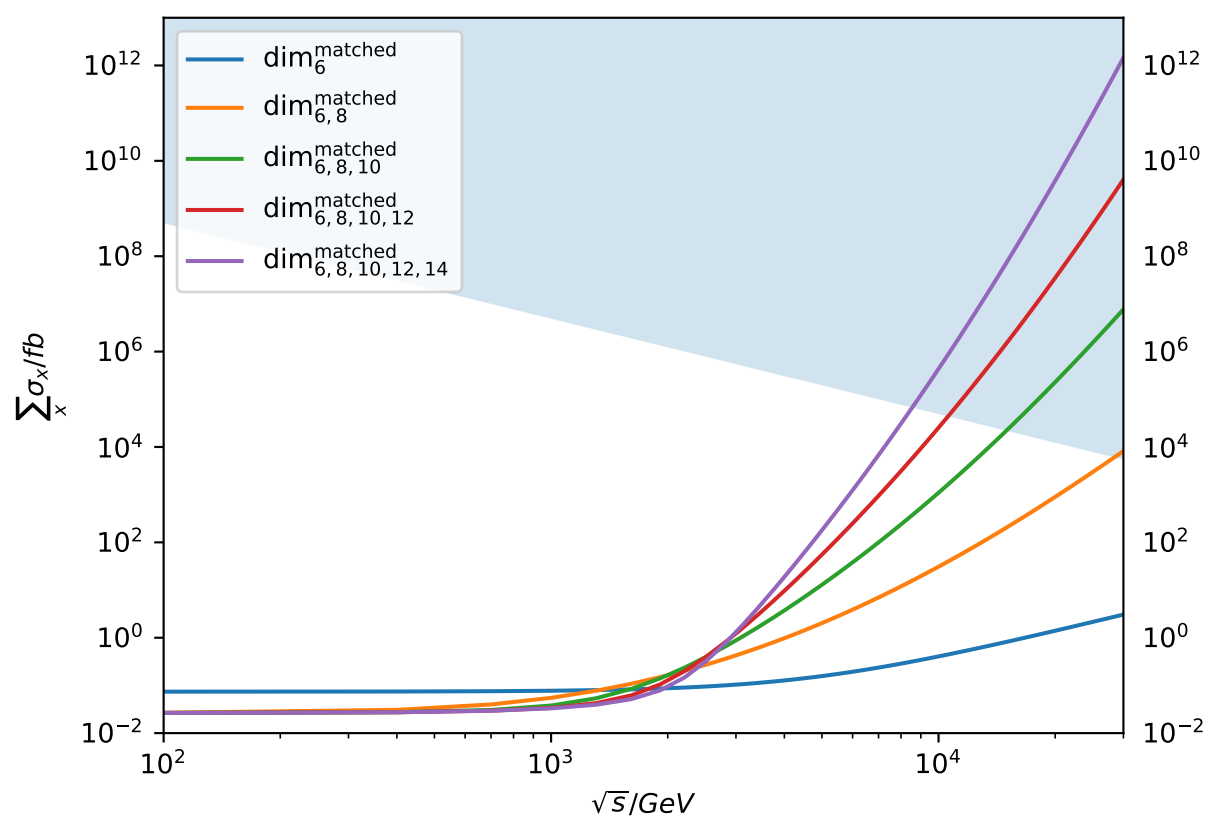

Figure 3. Inclusive inelastic cross section $\mu^{+} \mu^{-} \rightarrow X$ for multiple Goldstone and Higgs-boson production in the GBET approximation. We show the result for the sequence of SMEFT scenarios defined by the conditions (2.25), truncated at dimension $d=6,8,10,12,14$, respectively. The maximal multiplicity of the final state is $n=3,5,7,9,11$, respectively. The shaded area indicates the region that is excluded by the universal unitarity bound for the inclusive cross section (2.33).

numeric values of the unitarity bound are $95 \mathrm{TeV}, 17 \mathrm{TeV}$, and $11 \mathrm{TeV}$, respectively. For $d \geq 8$, the values of these bounds lie within the energy range that is accessible at a future muon collider. They imply large amounts of observable multi-boson production. The strong suppression of the corresponding SM processes enables a study already significantly below those upper bounds. Furthermore, we expect observable effects even if only a fraction of the muon mass is due to the new-physics contributions that are parameterized by those operators.

In the previous subsection, we have discussed an analogous sequence of phenomenological scenarios within the SMEFT framework, where we require that local Higgs-fermion couplings are absent up to a given Higgs multiplicity $n$. This requirement enforces a specific choice of the SMEFT operator coefficients $C_{\ell \varphi}^{(n)}$ up to dimension $d=2 n+4$, as defined by (2.25). The limit $d \rightarrow \infty$ corresponds to the case of no local Higgs-fermion couplings of any multiplicity. We emphasize that this peculiar choice is merely an extreme case of a generic anomalous muon Yukawa sector. The generic case is parameterized within the SMEFT or HEFT formalisms, allowing the coefficients of the higher-dimensional couplings to vary freely within the constraints imposed by unitarity.

In quantitative terms, the unitarity constraint for the total inelastic cross section $\sigma_{\mu^{+} \mu^{-} \rightarrow X}(s)$, where $X \neq \mu^{+} \mu^{-}$, is given by the inequality

$$
\sum_{X} \sigma_{\mu^{+} \mu^{-} \rightarrow X}(s) \leq \frac{4 \pi}{s}
$$


In figure 3 we display the total cross section for this sequence of scenarios, including operators up to dimension $d=6,8,10, \ldots$ and compare it with the upper bound (2.33). The cross section has been evaluated using the GBET, summing over all final states. The SM contribution $(d=4)$ can be neglected for this purpose, and the boson masses are set to zero. The multiplicity of the Higgs and Goldstone bosons extends up to $n=d-3$, which evaluates to $n=3,5,7, \ldots$, respectively.

We observe that for $d \leq 10$ (i.e., $n \leq 7$ ), the sum over cross sections does not touch the unitarity bound before $15 \mathrm{TeV}$, while for higher dimension and multiplicity, the curves cross already at collider energies within the range considered for a muon collider. In the $d \rightarrow \infty$ case, the multiplicity of extra Goldstone-boson production becomes unbounded, and the unitarity limit for the sequence of scenarios (2.25) formally drops towards the original electroweak scale [84]. Even if we account for finite vector-boson masses, such a scenario should be qualified as strongly interacting, and finite-order predictions in the multi-TeV range become invalid. Of course, we do not expect the actual operator coefficients to strictly follow such a pattern, so the argument should rather be understood as a guideline regarding the inherent limitations of the EFT in the current context.

For this reason, we consider lower-dimensional operators in the SMEFT or HEFT expansions individually. The presence of extra Higgs bosons in the gauge-invariant SMEFT operators of fixed dimension delays the potential onset of new (strong) interactions to higher energy. While in the tables and plots of the subsequent sections we will frequently refer to the $d=\infty$ limit for illustration, in our phenomenological study we work with HiggsGoldstone multiplicities $n \leq 4$ and limit the dimensions of the included SMEFT operators to $d=6,8,10$. For those final states, figure 3 indicates that unitarity is not yet relevant at a muon collider as proposed, even if we adopt one of the extreme scenarios described above. Clearly, higher multiplicities may yield even stronger effects, but their contributions depend on further coefficients in the EFT expansion and should therefore be regarded as model-dependent. In fact, if in (2.33) we restrict the sum over final states to $n \leq 4$, there is no problem with unitarity for any of the parameter sets shown in figure 3 . The numerical results of our study below will rely on the lowest multiplicities and analyze small deviations from the SM where the actual effect is at the limit of the collider sensitivity, orders of magnitude below the unitarity bound.

\subsubsection{Multi-boson production and cross section ratios}

Obviously, the most direct and model-independent probe to the muon-Higgs coupling would be the $s$-channel resonant production

$$
\mu^{+} \mu^{-} \rightarrow H .
$$

This was the motivation for a muon-collider Higgs factory [85, 86]. This process would put an extremely high demand on the collider beam quality to resolve the narrow width of the Higgs boson, and on the integrated luminosity. Off the resonance at higher energies, one could consider to study this coupling by utilizing the process of radiative return [87]. Although the expected cross sections for multiple Higgs production $\mu^{+} \mu^{-} \rightarrow H H$ and 
$H H H$ are quite small as shown later, they receive a power enhancement $E / \Lambda$ of the effective coupling of $\kappa_{\mu}$, if a new interaction like the dimension- 6 operator, eq. (2.28), is present. If an analogous dimension-8 operator is present with a Wilson coefficient $c_{\ell \varphi}^{(2)} \sim 1 / \Lambda^{4}$, the physical muon mass and the Yukawa couplings are given by

$$
\begin{aligned}
& m_{\mu}^{(8)}=\frac{v}{\sqrt{2}}\left(y_{\mu}-\frac{v^{2}}{2} c_{\ell \varphi}^{(1)}-\frac{v^{4}}{4} c_{\ell \varphi}^{(2)}\right), \\
& \lambda_{\mu}^{(8)}=\left(y_{\mu}-\frac{3 v^{2}}{2} c_{\ell \varphi}^{(1)}-\frac{5 v^{4}}{4} c_{\ell \varphi}^{(2)}\right),
\end{aligned}
$$

The dimension- 8 operator causes a rise of $n$-boson production cross sections, and ultimately a saturation of tree-level unitarity, for up to $n=5$ as discussed in the previous section. Depending on the relative size of the individual contributions at a given energy, the ratios of individual multi-boson channels are determined by either $Y_{e}, C_{\ell \varphi}^{(1)}$ or $C_{\ell \varphi}^{(2)}$. Final states with more Higgs bosons receive direct contributions which rapidly rise with energy $(E / \Lambda)^{n}$.

The operators introduced in eqs. (2.23) and (2.34)-(2.35) induce contact terms, schematically written as,

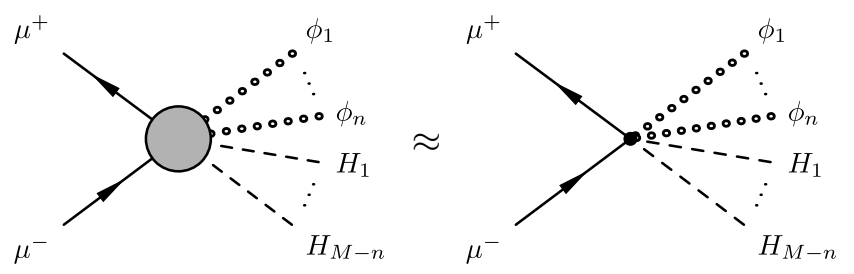

which are dominant in the high-energy limit as there is no suppression in $\sqrt{s}$ from propagator denominators. Let us denote the Feynman rules for a multi-boson final state $X$ as

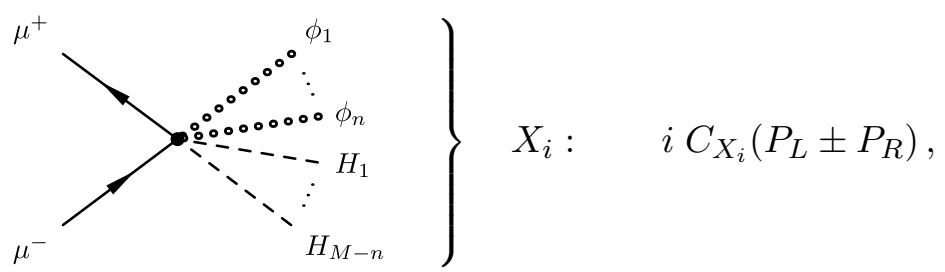

where $C_{X_{i}}$ is a linear combination of Wilson coefficients, and $i$ labels all possible final states for a given multiplicity. The sign in $\left(P_{L} \pm P_{R}\right)$ depends on the number of Goldstone bosons $\phi^{0}$ in the final state and does not play any role for the following argument. The spin-averaged matrix element reads $\left(k_{i}, i=1,2\right.$ are the two muon momenta, $s=2 k_{1} \cdot k_{2}$, where we ignored the muon mass in the kinematics of the matrix element)

$$
\begin{aligned}
\overline{\left|\mathcal{A}_{X_{i}}\right|^{2}} & =\frac{1}{4}\left|C_{X_{i}}\right|^{2} \sum_{s_{1}, s_{2}} \bar{v}_{s_{1}}\left(k_{1}\right)\left(P_{L} \pm P_{R}\right) u_{s_{2}}\left(k_{2}\right) \bar{u}_{s_{2}}\left(k_{2}\right)\left(P_{R} \pm P_{L}\right) v_{s_{1}}\left(k_{1}\right) \\
& =\left|C_{X_{i}}\right|^{2} \times\left(k_{1} \cdot k_{2} \mp m_{\mu}^{2}\right) \approx \frac{\left|C_{X_{i}}\right|^{2} s}{2} .
\end{aligned}
$$

As the spin-averaged matrix element in that approximation is constant, the integration over the phase space is trivial and yields a cross section

$$
\sigma^{X_{i}}=\frac{(2 \pi)^{4}}{2 s}\left|\mathcal{A}_{X_{i}}\right|^{2}\left(\prod_{j \in J_{X_{i}}} \frac{1}{n_{j} !}\right) \Phi_{M}\left(k_{1}+k_{2} ; p_{1}, \ldots, p_{M}\right),
$$




\begin{tabular}{|c|c|c|c|c|c|c|}
\hline & \multicolumn{5}{|c|}{$\Delta \sigma^{X} / \Delta \sigma^{W^{+} W^{-}}$} \\
\hline & \multicolumn{5}{|c|}{ SMEFT } & \multicolumn{2}{c|}{ HEFT } \\
\hline$X$ & $\operatorname{dim}_{6}$ & $\operatorname{dim}_{8}$ & $\operatorname{dim}_{6,8}$ & $\operatorname{dim}_{6,8}^{\text {matched }}$ & $\operatorname{dim}_{\infty}$ & $\operatorname{dim}_{\infty}^{\text {matched }}$ \\
\hline$W^{+} W^{-}$ & 1 & 1 & 1 & 1 & 1 & 1 \\
$Z Z$ & $1 / 2$ & $1 / 2$ & $1 / 2$ & $1 / 2$ & $1 / 2$ & $1 / 2$ \\
\hline$Z H$ & 1 & $1 / 2$ & 1 & 1 & $R_{(2), 1}^{\text {HEFT }}$ & 1 \\
$H H$ & $9 / 2$ & $25 / 2$ & $R_{(2), 1}^{\text {SMEFT }} / 2$ & 0 & $2 R_{(2), 2}^{\text {HEFT }}$ & 0 \\
\hline
\end{tabular}

Table 1. Ratios of final-state cross-section deviations in diboson production, assuming that the leading muon-Yukawa contribution originates from various combinations of $d=6$ and $d=8$ operators in SMEFT, or from a direct contribution in the HEFT, respectively. The term "matched" indicates the matching to a model with a vanishing muon-Yukawa coupling. See the text for details. The coefficients $R_{(2), i}$ are defined in (2.39).

where $\Phi_{M}^{X_{i}}\left(k_{1}+k_{2} ; p_{1}, \ldots, p_{M}\right)$ is the $M$-particle phase-space volume and $J_{X_{i}}$ is the set of indistinguishable particles $X_{i}$ in the final state with numbers $n_{j}$ for particle $j \in J_{X^{i}}$. As we study the limit of very high energies, we neglect all particle masses, and the phasespace volume will be the same for all final states $X_{i}$. In the center-of-mass (CMS) system (cf. [88]), the $M$-particle phase space is given by ( $\Gamma$ is the Euler gamma function)

$$
\Phi_{M}^{X_{i}}\left(k_{1}+k_{2} ; p_{1}, \ldots, p_{M}\right)=\frac{1}{(2 \pi)^{3 M}}\left(\frac{\pi}{2}\right)^{M-1} \frac{s^{M-2}}{\Gamma(M) \Gamma(M-1)} .
$$

In order to study the effects from specific operator coefficients, it is beneficial to look into ratios of cross sections with respect to a certain reference cross section for a specific exclusive final state of the same multiplicity. For such cross-section ratios we find

$$
R^{X_{i}}:=\frac{\sigma^{X_{i}}}{\sigma^{X_{\mathrm{ref}}}}=\frac{\left|C_{X_{i}}\right|^{2}\left(\prod_{j \in J_{X_{i}}} \frac{1}{n_{j} !}\right)}{\left|C_{X_{\mathrm{ref}}}\right|^{2}\left(\prod_{j \in J_{X_{\mathrm{ref}}}} \frac{1}{n_{j} !}\right)} .
$$

In the following, we discuss ratios of deviations of production cross sections from their SM values for final-state multiplicities $n=2,3,4$. For each multiplicity, the cross-section deviations $\Delta \sigma^{X}$ for different final states $X$ will be normalized with respect to a particular exclusive reference final state, which is $W^{+} W^{-}$for dibosons, $W^{+} W^{-} H$ for tribosons, and $W^{+} W^{-} H H$ for four bosons, respectively. The cross sections are calculated in the GBET approximation for massless Goldstone bosons; for longitudinal $W^{ \pm}$and $Z$ boson final states they become exact in the limit that both their masses as well as the SM contributions to these cross sections can be neglected. We are considering these ratios for different EFT scenarios, namely for truncating the SMEFT series of higher-dimensional operators at dimension $d=6,8,10$, respectively, as well as for the non-linear HEFT case.

In detail, in table 1 we consider the diboson final states for the cases of a pure $d=6$ contribution $\left(\operatorname{dim}_{6}\right)$, a pure $=8$ contribution $\left(\operatorname{dim}_{8}\right)$, a mixed contribution $\left(\operatorname{dim}_{6,8}\right)$, and for the case where the $d=6$ and $d=8$ operators are tuned to cancel the leading-order Yukawa coupling according to $(2.34),(2.35)$, denoted $\operatorname{dim}_{6,8}^{\text {matched }}$. For the non-linear HEFT 


\begin{tabular}{|c|c|c|c|c|c|c|}
\hline & \multicolumn{5}{|c|}{$\Delta \sigma^{X} / \Delta \sigma^{W^{+} W^{-} H}$} \\
\hline & \multicolumn{3}{|c|}{$\operatorname{SMEFT}$} & \multicolumn{2}{c|}{ HEFT } \\
\hline$\mu^{+} \mu^{-} \rightarrow X$ & $\operatorname{dim}_{6}$ & $\operatorname{dim}_{8}$ & $\operatorname{dim}_{6,8}$ & $\operatorname{dim}_{6,8}^{\text {matched }}$ & $\operatorname{dim}_{\infty}$ & $\operatorname{dim}_{\infty}^{\text {matched }}$ \\
\hline$W W Z$ & 1 & $1 / 9$ & $R_{(3), 1}^{\mathrm{SMEFT}}$ & $1 / 4$ & $R_{(3), 1}^{\mathrm{HEFT}} / 9$ & $1 / 4$ \\
$Z Z Z$ & $3 / 2$ & $1 / 6$ & $3 R_{(3), 1}^{\mathrm{SMET}} / 2$ & $3 / 8$ & $R_{(3), 1}^{\mathrm{HEFT}} / 6$ & $3 / 8$ \\
\hline$W W H$ & 1 & 1 & 1 & 1 & 1 & 1 \\
$Z Z H$ & $1 / 2$ & $1 / 2$ & $1 / 2$ & $1 / 2$ & $1 / 2$ & $1 / 2$ \\
$Z H H$ & $1 / 2$ & $1 / 2$ & $1 / 2$ & $1 / 2$ & $2 R_{(3), 2}^{\mathrm{HEFT}}$ & $1 / 2$ \\
$H H H$ & $3 / 2$ & $25 / 6$ & $3 R_{(3), 2}^{\mathrm{SMEFT}} / 2$ & $75 / 8$ & $6 R_{(3), 3}^{\mathrm{HEFT}}$ & 0 \\
\hline
\end{tabular}

Table 2. Same as table 1 but for triboson production. The coefficients $R_{(3), i}$ are listed in $(2.40)-(2.41)$.

setup, the first column $\left(\operatorname{dim}_{\infty}\right)$ takes into account the full tower, in principle, though only the lowest dimension contributes at tree level due to the $n$-arity of the vertex. The last column ( $\operatorname{dim}_{\infty}^{\text {matched }}$ ) is the matched case again with a vanishing Yukawa coupling, calculated by taking into account a sufficiently large number of terms corresponding to the linear setup. The list of processes includes direct production of up to two Higgs bosons. The non-rational coefficients in this and the following tables are expressed in terms of ratio coefficients, $R_{(N), i}^{\mathrm{HEFT} / \mathrm{SMEFT}}$, where $N$ is the multiplicity of the boson final state, and $i$ labels the contribution from higher-dimensional operators to the given multiplicity with increasing operator order,

$$
R_{(2), 1}^{\mathrm{SMEFT}}=\left(\frac{5 v^{2} c_{\ell \varphi}^{(2)}+c_{\ell \varphi}^{(1)}}{v^{2} c_{\ell \varphi}^{(2)}+c_{\ell \varphi}^{(1)}}\right)^{2}, \quad R_{(2), 1}^{\mathrm{HEFT}}=\left(\frac{y_{1}}{y_{\mu}}\right)^{2}, \quad R_{(2), 2}^{\mathrm{HEFT}}=\left(\frac{y_{2}}{y_{\mu}}\right)^{2}
$$

Here, the $c_{\ell \varphi}^{(i)}$ operator coefficients of SMEFT have been introduced above in (2.34), (2.35), while by $y_{i}$ we have denoted the Yukawa couplings of the muon to $i+1$ Higgs bosons in the HEFT parameterization. In SMEFT, if the $\operatorname{dim}_{6}$ contributions dominate, then $R^{\mathrm{SMEFT}} \sim 1$. On the other hand, the $\operatorname{dim}_{8}$ contributions can modify this behavior. In HEFT, $R^{\mathrm{HEFT}}$ could be larger than 1 in a strongly coupled theory. In addition, those anomalous contributions will lead to enhancements at high energies.

The cross-section ratios in the case of triboson production are summarized in table 2 . Here, all exclusive final-state production cross sections are normalized to the $W^{+} W^{-} H$ final state, which is the one whose phenomenology we will study in detail in section 3. As for the case of diboson production, we consider scenarios with a pure $d=6$ contribution $\left(\operatorname{dim}_{6}\right)$, a pure $d=8$ contribution $\left(\operatorname{dim}_{8}\right)$, a mixed contribution $\left(\operatorname{dim}_{6,8}\right)$, and for the case where the $d=6$ and $d=8$ operators are tuned to cancel the leading-order Yukawa coupling according to $(2.34),(2.35)$ ( $\left.\operatorname{dim}_{6,8}^{\text {matched}}\right)$, respectively. Exclusive final states contain up to three physical Higgs bosons. For the triboson case, we define the following ratio coefficients for the SMEFT and HEFT case, respectively, as

$$
R_{(3), 1}^{\mathrm{SMEFT}}=\left(\frac{v^{2} c_{\ell \varphi}^{(2)}+c_{\ell \varphi}^{(1)}}{3 v^{2} c_{\ell \varphi}^{(2)}+c_{\ell \varphi}^{(1)}}\right)^{2}, \quad R_{(3), 2}^{\mathrm{SMEFT}}=\left(\frac{5 v^{2} c_{\ell \varphi}^{(2)}+c_{\ell \varphi}^{(1)}}{3 v^{2} c_{\ell \varphi}^{(2)}+c_{\ell \varphi}^{(1)}}\right)^{2}
$$




\begin{tabular}{|c|c|c|c|c|c|c|}
\hline & \multicolumn{6}{|c|}{$\Delta \sigma^{X} / \Delta \sigma^{W W H H}$} \\
\hline & \multicolumn{4}{|c|}{ SMEFT } & \multicolumn{2}{|c|}{ HEFT } \\
\hline$\mu^{+} \mu^{-} \rightarrow X$ & $\operatorname{dim}_{6,8}$ & $\operatorname{dim}_{10}$ & $\operatorname{dim}_{6,8,10}$ & $\operatorname{dim}_{6,8,10}^{\text {matched }}$ & $\operatorname{dim}_{\infty}$ & $\operatorname{dim}_{\infty}^{\text {matched }}$ \\
\hline$W W W W$ & $2 / 9$ & $2 / 25$ & $2 R_{(4), 1}^{\mathrm{SMEFT}} / 9$ & $1 / 2$ & $R_{(4), 1}^{\mathrm{HEFT}} / 18$ & $1 / 2$ \\
\hline$W W Z Z$ & $1 / 9$ & $1 / 25$ & $R_{(4), 1}^{\mathrm{SMEFT}} / 9$ & $1 / 4$ & $R_{(4), 1}^{\mathrm{HEFT} / 36}$ & $1 / 4$ \\
\hline$Z Z Z Z$ & $1 / 12$ & $3 / 100$ & $R_{(4), 1}^{\mathrm{SMEFT}} / 12$ & $3 / 16$ & $R_{(4), 1}^{\mathrm{HEFT}} / 48$ & $3 / 16$ \\
\hline$W W Z H$ & $2 / 9$ & $2 / 25$ & $2 R_{(4), 1}^{\mathrm{SMEFT}} / 9$ & $1 / 2$ & $R_{(4), 2}^{\mathrm{HEFT}} / 8$ & $1 / 2$ \\
\hline$W W H H$ & 1 & 1 & 1 & 1 & 1 & 1 \\
\hline$Z Z Z H$ & $1 / 3$ & $3 / 25$ & $R_{(4), 1}^{\mathrm{SMEFT}} / 3$ & $3 / 4$ & $R_{(4), 2}^{\mathrm{HEFT}} / 12$ & $3 / 4$ \\
\hline$Z Z H H$ & $1 / 2$ & $1 / 2$ & $1 / 2$ & $1 / 2$ & $1 / 2$ & $1 / 2$ \\
\hline$Z H H H$ & $1 / 3$ & $1 / 3$ & $1 / 3$ & $1 / 3$ & $3 R_{(4), 3}^{\mathrm{HEFT}}$ & $1 / 3$ \\
\hline$H H H H$ & $25 / 12$ & $49 / 12$ & $25 R_{(4), 2}^{\mathrm{SMEFT}} / 12$ & $1225 / 48$ & $12 R_{(4), 4}^{\mathrm{HEFT}}$ & 0 \\
\hline
\end{tabular}

Table 3. Same as tables 1 and 2 but for four-boson production. The coefficients $R_{(4), i}$ are listed in $(2.42)-(2.43)$.

and

$$
R_{(3), 1}^{\mathrm{HEFT}}=\left(\frac{y_{\mu}}{y_{1}}\right)^{2}, \quad R_{(3), 2}^{\mathrm{HEFT}}=\left(\frac{y_{2}}{y_{1}}\right)^{2}, \quad R_{(3), 3}^{\mathrm{HEFT}}=\left(\frac{y_{3}}{y_{1}}\right)^{2} .
$$

We recall that at multiplicity $n=4$ and beyond, the dimension-6 SMEFT operator does not directly contribute in the GBET approximation, so we choose to include the effects of the analogous dimension- 8 and dimension-10 operators in the table for the production of quartic final states. In table 3, we display the ratios of four-particle final state cross sections; definitions and conventions are analogous to those in table 2. The ratio coefficients for the four-boson final states are given by

$$
R_{(4), 1}^{\mathrm{SMEFT}}=\left(\frac{3 v^{2} c_{\ell \varphi}^{(3)}+2 c_{\ell \varphi}^{(2)}}{5 v^{2} c_{\ell \varphi}^{(3)}+2 c_{\ell \varphi}^{(2)}}\right)^{2}, \quad R_{(4), 2}^{\mathrm{SMEFT}}=\left(\frac{7 v^{2} c_{\ell \varphi}^{(3)}+2 c_{\ell \varphi}^{(2)}}{5 v^{2} c_{\ell \varphi}^{(3)}+2 c_{\ell \varphi}^{(2)}}\right)^{2}
$$

and

$$
R_{(4), 1}^{\mathrm{HEFT}}=\left(\frac{y_{\mu}}{y_{2}}\right)^{2}, \quad R_{(4), 2}^{\mathrm{HEFT}}=\left(\frac{y_{1}}{y_{2}}\right)^{2}, \quad R_{(4), 3}^{\mathrm{HEFT}}=\left(\frac{y_{3}}{y_{2}}\right)^{2}, \quad R_{(4), 4}^{\mathrm{HEFT}}=\left(\frac{y_{4}}{y_{2}}\right)^{2} .
$$

To numerically cross check the analytical results for the cross-section ratios, we implemented the extreme case of the SM with a vanishing as well as with a $\kappa$-rescaled muon Yukawa coupling, respectively, within the same Monte Carlo (MC) framework that we used for our phenomenological study in section 3 for multi-boson final states $X_{i}$ for the class of processes $\mu^{+} \mu^{-} \rightarrow W^{+} W^{-} H^{M-2}$. Our numerical MC results agree perfectly with the ratios given in tables 1,2 , and 3 , thereby validating our SMEFT implementation.

In summary, the common feature of all versions of the modified Yukawa sector is a proliferation of multi-boson production at high energy. The anomalous contributions do 


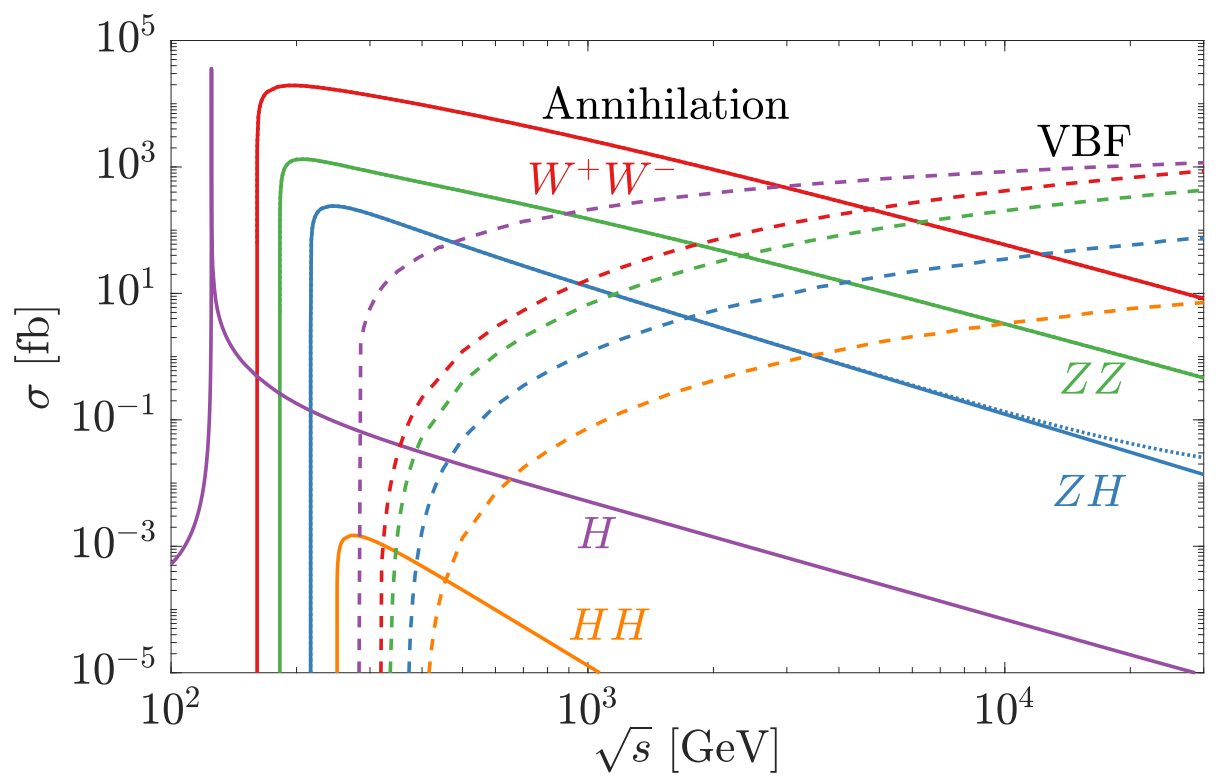

Figure 4. The cross sections of diboson production at a $\mu^{+} \mu^{-}$collider as a function of the c.m. energy $\sqrt{s}$. The solid and dotted lines are for the direct annihilation with muon Yukawa coupling as $\kappa_{\mu}=1$ and $\kappa_{\mu}=0$ (2) (hardly visible), respectively. The dashed rising curves are the (charged) vector boson fusions (VBF), $\mu^{+} \mu^{-} \rightarrow \nu_{\mu} \bar{\nu}_{\mu} X$, calculated using the fixed-order (FO) approach with a cut on the invariant mass of $\nu_{\mu} \bar{\nu}_{\mu}$ pair $M_{\nu_{\mu} \bar{\nu}_{\mu}}>150 \mathrm{GeV}$. All calculations are carried out with WHIZARD 2.8.5.

not interfere with SM production due to the mismatch in helicity. The dimensionality of the anomalous interactions determines the particle multiplicity in the energy range where the new interactions start to dominate over SM particle production. The breakdown into distinct final states allows for drawing more detailed conclusions on the operator content and thus the underlying mechanism.

In the next section, we are studying the phenomenology of such a SMEFT setup featuring a modified muon Yukawa coupling and assess our sensitivity to it at a highenergy $\mu^{+} \mu^{-}$collider, using the paradigm process $\mu^{+} \mu^{-} \rightarrow W^{+} W^{-} H$. Processes with multiple Higgs bosons only in the final state are also very interesting and may yield further strong signals, as can be read off from the tables above. The SM rates for those final states are tiny, so any signal is a clear indication for new physics in this sector. However, the cross sections of pure multi-Higgs final states such as $H H H$ are also more model-dependent. By adjusting the higher-order coefficients in the SMEFT expansion, those cross sections can be varied at will without altering the ordinary muon Yukawa coupling. This is evident since in the alternative HEFT formalism where the Higgs is a singlet, the local couplings to different numbers of Higgs bosons are not related at all, cf. section 2.2.1. Turning the argument around, if an anomalous Goldstone-boson signal is found as we study below, analyzing the relative magnitude of pure-Higgs final states will reveal details about the underlying Higgs-sector dynamics. We defer this to a separate phenomenological study. 


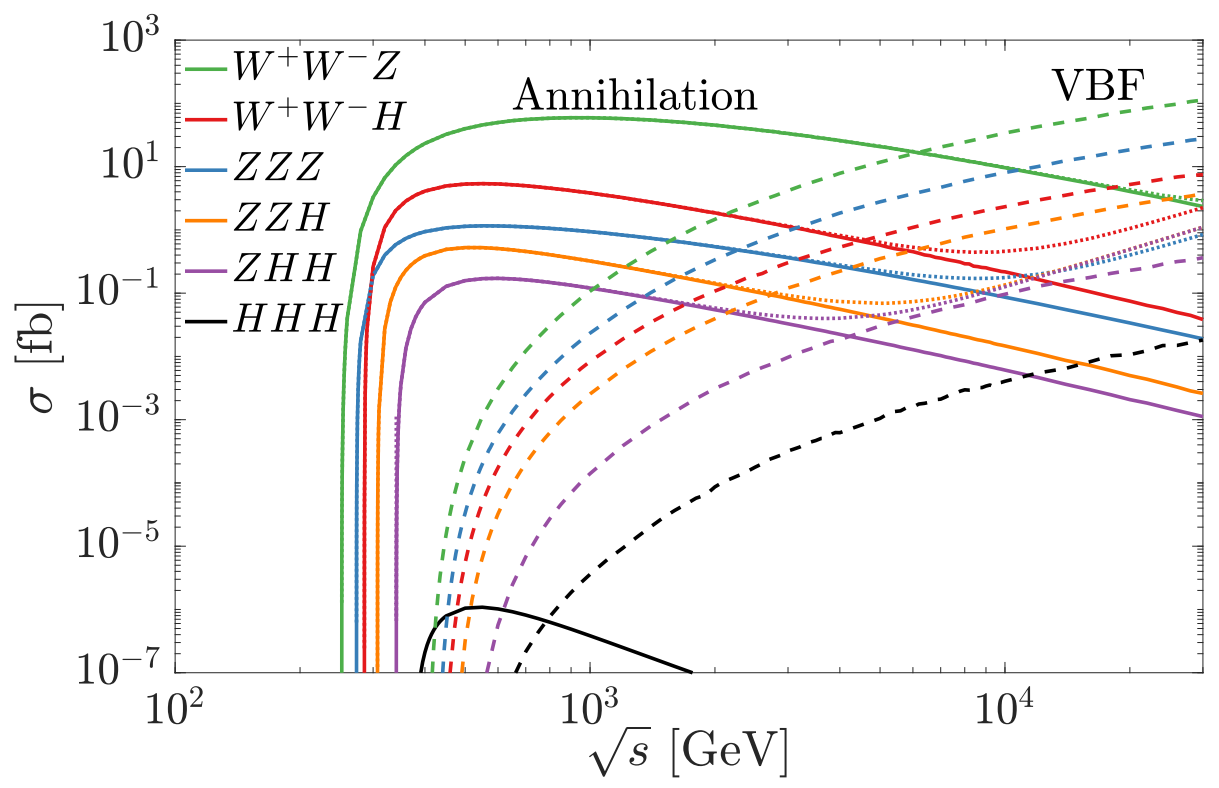

Figure 5. Similar to figure 4 , the cross sections of three-boson production at a $\mu^{+} \mu^{-}$collider as a function of the c.m. energy $\sqrt{s}$.

\section{Phenomenology of muon-Higgs coupling at a high-energy muon col- lider}

In this section, we explore the phenomenology of multi-boson production for the sensitivity to the muon Yukawa coupling at a muon collider with collision energy in the range $1<\sqrt{s}<$ $30 \mathrm{TeV}$, with an integrated luminosity, which scales with energy quadratically as [21, 22],

$$
\mathcal{L}=\left(\frac{\sqrt{s}}{10 \mathrm{TeV}}\right)^{2} 10 \mathrm{ab}^{-1}
$$

\subsection{Multi-boson production}

To numerically determine the different multi-boson production cross sections and later on assess the sensitivity to the muon Yukawa coupling, we parameterize the EFT contributions discussed in the last section with a model-independent coupling $\kappa_{\mu}$, e.g., eq. (2.20) or (2.30), and implement it into the multi-purpose event generator WHIZARD 2.8.5 [89-91] using its plugin to external models [92]. This is building upon the EFT frameworks used for multi-boson production and vector-boson scattering at hadron [93-96] and electronpositron colliders $[97,98]$, which we adapted here for the muon collider. The QED initialstate radiation (ISR), resummed to all orders in soft photons and up to third order in hard-collinear radiation, is equally applicable to the muon collider. Beam spectra for multi-TeV muon colliders are much less complicated than for electron-positron colliders and can be easily described with a Gaussian beam spread of $0.1 \%$. They are, however, not relevant at the level of this study.

In figures 4, 5 and 6, we first present the Standard Model (with $m_{\mu}=y_{\mu} v / \sqrt{2}$ ) cross sections for the production of two, three and four bosons, respectively, including the Higgs 


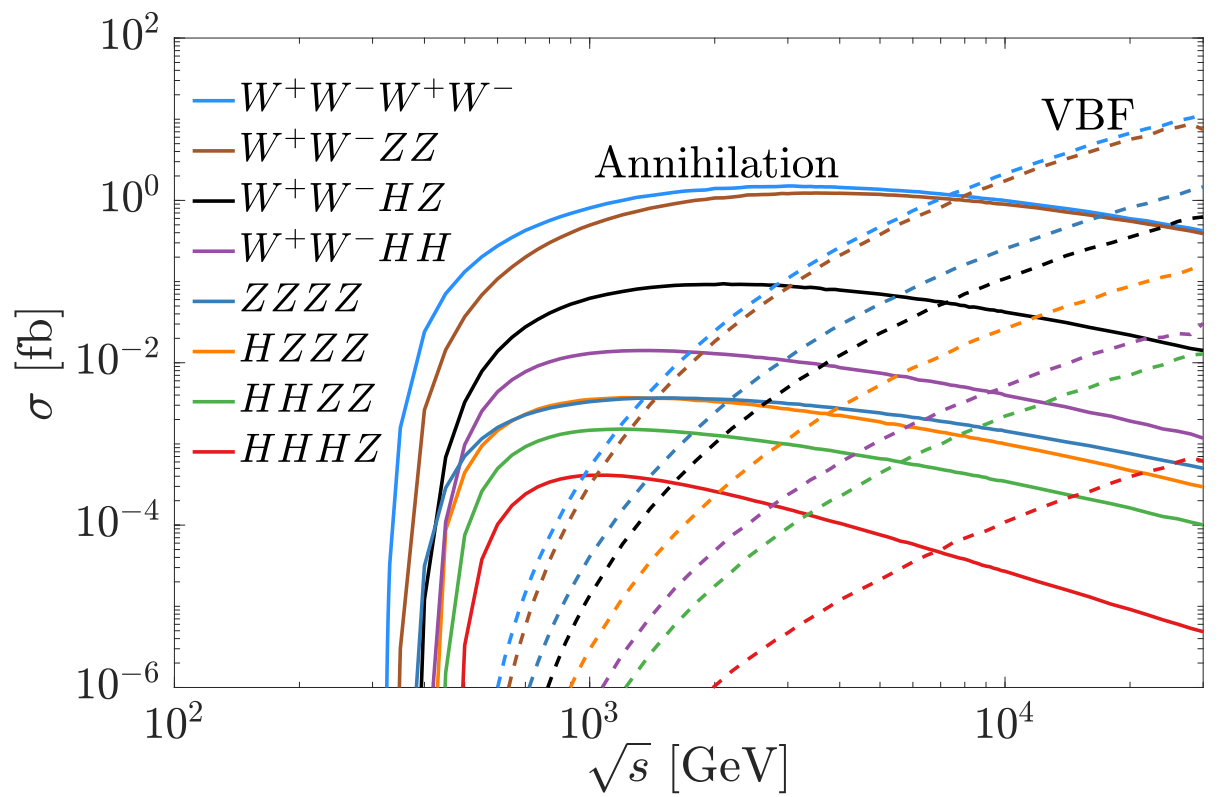

Figure 6. Similar to figure 4 , the cross sections of four-boson production at a $\mu^{+} \mu^{-}$collider as a function of the c.m. energy $\sqrt{s}$, for SM $\kappa_{\mu}=1$ only.

and the EW gauge bosons. The cross sections — in each case decreasing in size — are for two-boson production,

$$
W W, Z Z, Z H, H H
$$

for three-boson production,

$$
W W Z, W W H, Z Z Z, Z Z H, Z H H, H H H
$$

and for four-boson production,

$W W W W, W W Z Z, W W H Z, W W H H, Z Z Z Z, H Z Z Z, H H Z Z, H H H Z$

respectively. The single Higgs $(H)$ production is also illustrated in figure 4, which are obtained through $\mu^{+} \mu^{-} \rightarrow H$ recoiled by ISR. We present two classes of production mechanisms, namely, the direct $\mu^{+} \mu^{-}$annihilation and the vector boson fusion (VBF) resulting from the initial-state radiation off the muon beams. ${ }^{2}$ Representative Feynman diagrams for these production mechanisms are shown in figure 8 for the $W^{+} W^{-} H$ final state. Near the threshold, the annihilation cross sections dominate. With the increase of collision energy, they are suppressed by $1 / s$. The VBF mechanisms, on the other hand, increase with energy logarithmically $[25,26]$ and eventually take over above a few TeV. The $\mu^{+} \mu^{-}$

\footnotetext{
${ }^{2}$ If no specific indication, we only include the charged vector boson $\left(W^{ \pm}\right)$in VBF, i.e., $W^{+} W^{-} \rightarrow X$. The $Z$ boson fusion, $Z Z \rightarrow X$, is sub-leading due to its smaller vector coupling to leptons, with the example of $Z H H$ production demonstrated in table 4. The final states involving charged particles, e.g., $W^{+} W^{-} H$, can be produced through photon or photon- $Z$ fusion as well, which are mostly collinear to the initial beams. This background is largely excluded when a reasonable angular cut (e.g., $10^{\circ}<\theta<170^{\circ}$ ) is imposed, also illustrated in table 4.
} 

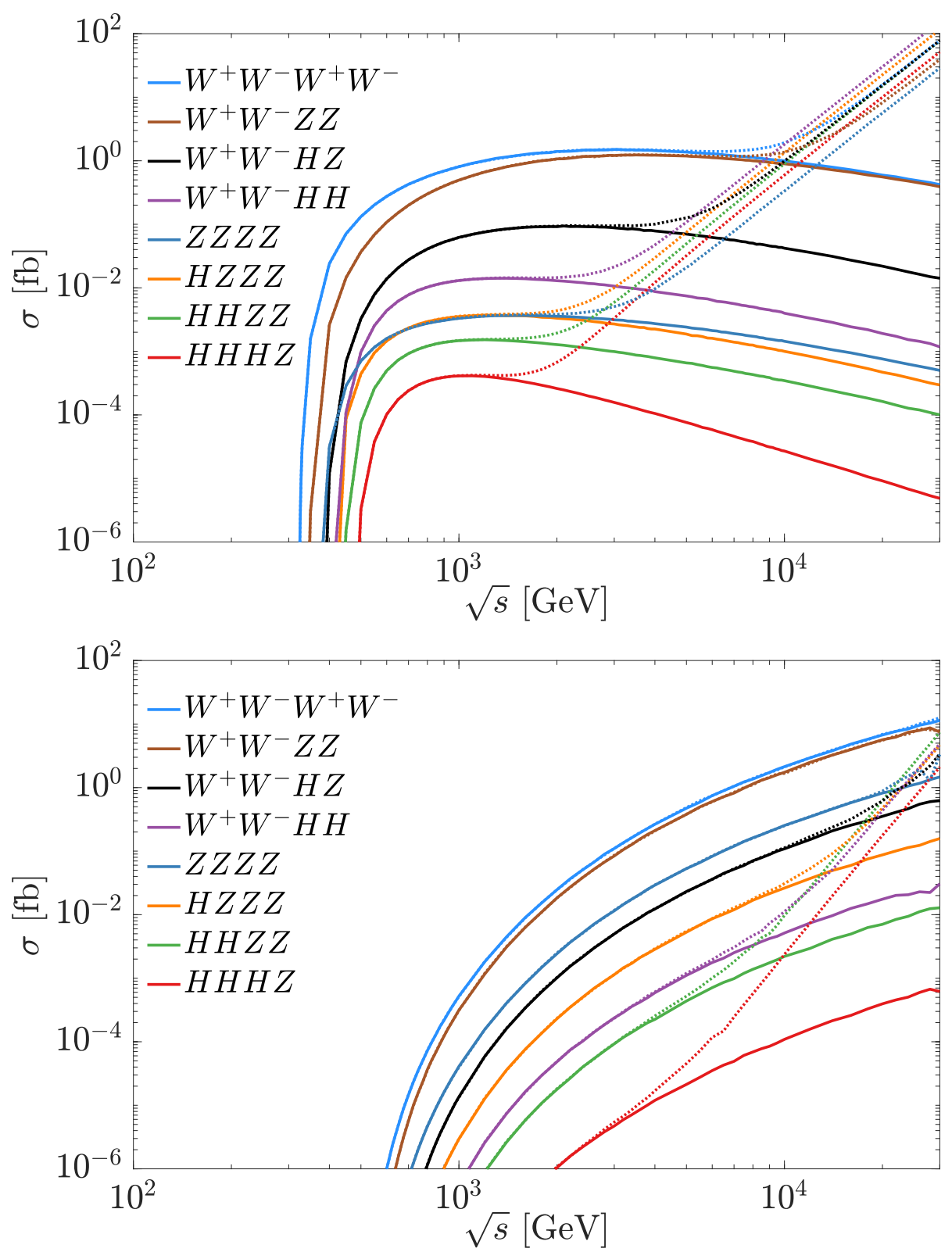

Figure 7. The cross sections of four-boson production at a $\mu^{+} \mu^{-}$collider via (a) annihilation $\mu^{+} \mu^{-} \rightarrow 4 B$ and (b) the (charged) vector boson fusions (VBF), $\mu^{+} \mu^{-} \rightarrow \nu_{\mu} \bar{\nu}_{\mu} X$ as functions of the c.m. energy $\sqrt{s}$. The solid and dotted lines are for the results with muon Yukawa coupling as $\kappa_{\mu}=1$ and $\kappa_{\mu}=0(2)$, respectively.

annihilation to multiple Higgs bosons is induced by the Yukawa and possible Higgs self interactions, while no gauge couplings. The corresponding cross sections are highly suppressed compared with the channels involving gauge boson(s), with examples of $H H$ and HHH demonstrated in figure 4 and 5. Therefore, there is no need to include four-Higgs production in eq. (3.4) or figure 7, and the corresponding phenomenological study of the pure Higgs production is largely left for the future. 
In the presence of anomalous couplings, the characteristic high-energy behavior shown in these figures is modified, as we discussed above in section 2. At asymptotically high energy, for each final state the new-physics contribution dominates over the SM and exhibits a simple and uniform power law as shown in figures 4, 5 and 7 by the dotted curves, which behave as straight lines in double-logarithmic plots.

In section 2 we provided a description within the EFT framework, in which the muon Yukawa coupling can receive contributions from new physics beyond the SM. The breakdown of the final states in terms of individual channels follows precisely the ratios of cross-section differences in tables 2 and 3, respectively, for the matched model. Given real data, measuring those ratios at various energy values will allow us to deduce the underlying pattern. In particular, the absence of pure multi-Higgs states is a special feature for the extreme scenario $d \rightarrow \infty$ which we used for the plots in figure 5 and 7 , i.e., there are no direct muon-Higgs couplings at any order. In a more generic scenario, multi-Higgs states will appear with a sizable rate, and the observable ratios of vector-boson and Higgs final states are related to the operator structure in the SMEFT expansion.

We now discuss the phenomenology of a modified muon Yukawa coupling in more detail. In the effective approach discussed above, the muon Yukawa coupling gets a modification like eq. (2.20) or (2.30). In such a way, $\kappa_{\mu}=1$ corresponds to the SM case. The deviation of $\kappa_{\mu}$ from 1 quantifies the new physics contribution, which serves as the signal in this work. In figures 5-7, we showed two such benchmark cross sections for $\kappa_{\mu}=0$ and 2 as dotted curves. They coincide with each other, which reflects a symmetry of the annihilation cross sections such that

$$
\left.\sigma\right|_{\kappa_{\mu}=1+\delta}=\left.\sigma\right|_{\kappa_{\mu}=1-\delta},
$$

where $\delta$ is the deviation from the SM muon Yukawa prediction, with an exception for the pure Higgs production.

With $\kappa_{\mu}=0(2)$ at a high energy, the annihilation cross sections of the $Z Z H$ and $Z H H$ channels merge in figure $5(\mathrm{a})$, which is a result of the Goldstone equivalence between the longitudinal $Z$ boson and the Higgs. A similar situation happens to the four-boson case at a higher collision energy in figure $7(\mathrm{~b})$. When compared with the Standard Model annihilation, we find that the $\kappa_{\mu}=0$ (2) cross sections agree at low collision energies, but gradually diverge as the collision energy increases. At $\sqrt{s}=30 \mathrm{TeV}$, the relative cross section deviation can be three orders of magnitude for the $Z H H$ case, while it amounts to $20 \%$ for $W W Z$ case. This big difference provides us a good opportunity to test the muon Yukawa coupling at a multi- $\mathrm{TeV} \mu^{+} \mu^{-}$collider.

As discussed above, and pointed out in [25, 26], the annihilation process, in our particular case here for three-boson production, is overcome at high energies by the vector-boson fusion (VBF) production which becomes dominant at all high-energy (lepton) colliders. Here we show the VBF cross sections as dashed lines in figure 5, as well. They are calculated with the fixed-order approach for fusion processes $\mu^{+} \mu^{-} \rightarrow \nu_{\mu} \bar{\nu}_{\mu} X$, where $X$ represents the desired final-state particles. We have imposed a cut on the invisible neutrinos, $M_{\nu_{\mu} \bar{\nu}_{\mu}}>150 \mathrm{GeV}[99,100]$, to suppress the on-shell decay $Z \rightarrow \nu_{\mu} \bar{\nu}_{\mu}$. We see that at an energy as high as $30 \mathrm{TeV}$, the VBF cross sections are generally $2 \sim 3$ magnitudes larger 


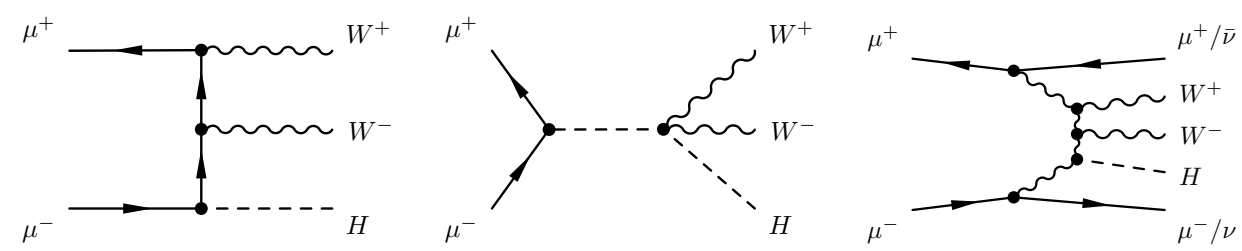

Figure 8. Representative diagrams for the signal annihilation process $\mu^{+} \mu^{-} \rightarrow W^{+} W^{-} H$ (left and middle), and for the VBF background process (right).
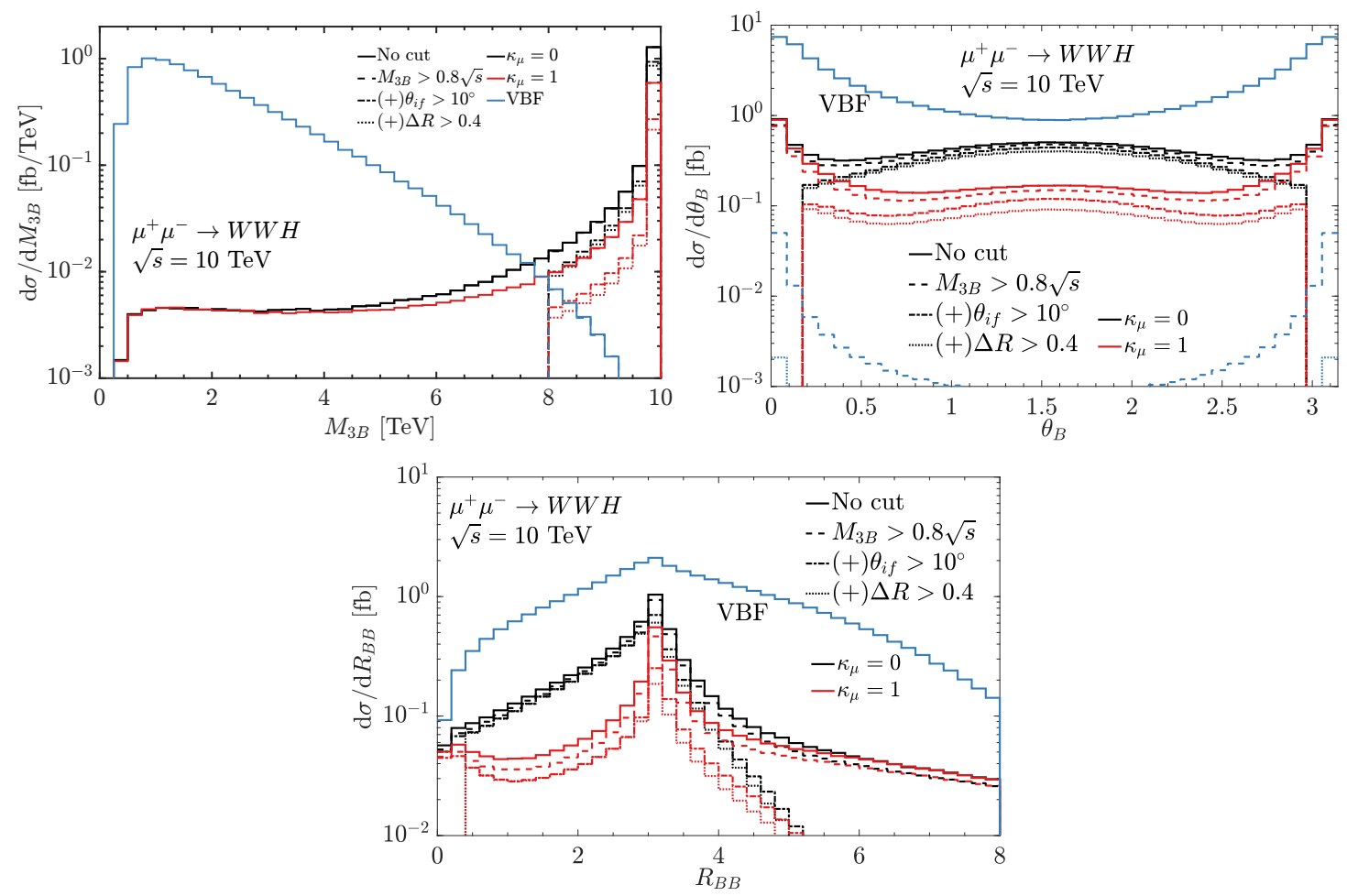

Figure 9. The kinematic distributions of the boson angle $\theta_{B}$, the diboson distance $R_{B B}$, and the triboson invariant mass $M_{3 B}(B=W, H)$, respectively, in the $W W H$ production at a $\sqrt{s}=10 \mathrm{TeV}$ $\mu^{+} \mu^{-}$collider.

than the annihilation processes for three-boson production. The relative size is even larger for the four-boson case. These channels will serve as backgrounds for the annihilation multi-boson productions when we measure the muon Yukawa coupling.

\subsection{Kinematic distributions}

As we know, the kinematic distributions for the annihilation and VBF processes behave very differently. We take the $W W H$ and $Z H H$ production at a $\sqrt{s}=10 \mathrm{TeV} \mu^{+} \mu^{-}$collider as benchmark examples ${ }^{3}$ and show the distributions of boson angles $\theta_{B}(B=W, Z, H)$, the diboson separation distances $R_{B B}=\sqrt{(\Delta \eta)^{2}+(\Delta \phi)^{2}}$ in the rapidity-azimuthal angle

\footnotetext{
${ }^{3}$ In triboson production, we choose $W W H$ as a demonstration example considering its large production rate, and $Z H H$ as another one for its relatively large deviation from the anomalous coupling. The $W W Z$ channel has an even larger cross section, while it suffers from a small relative deviation.
} 

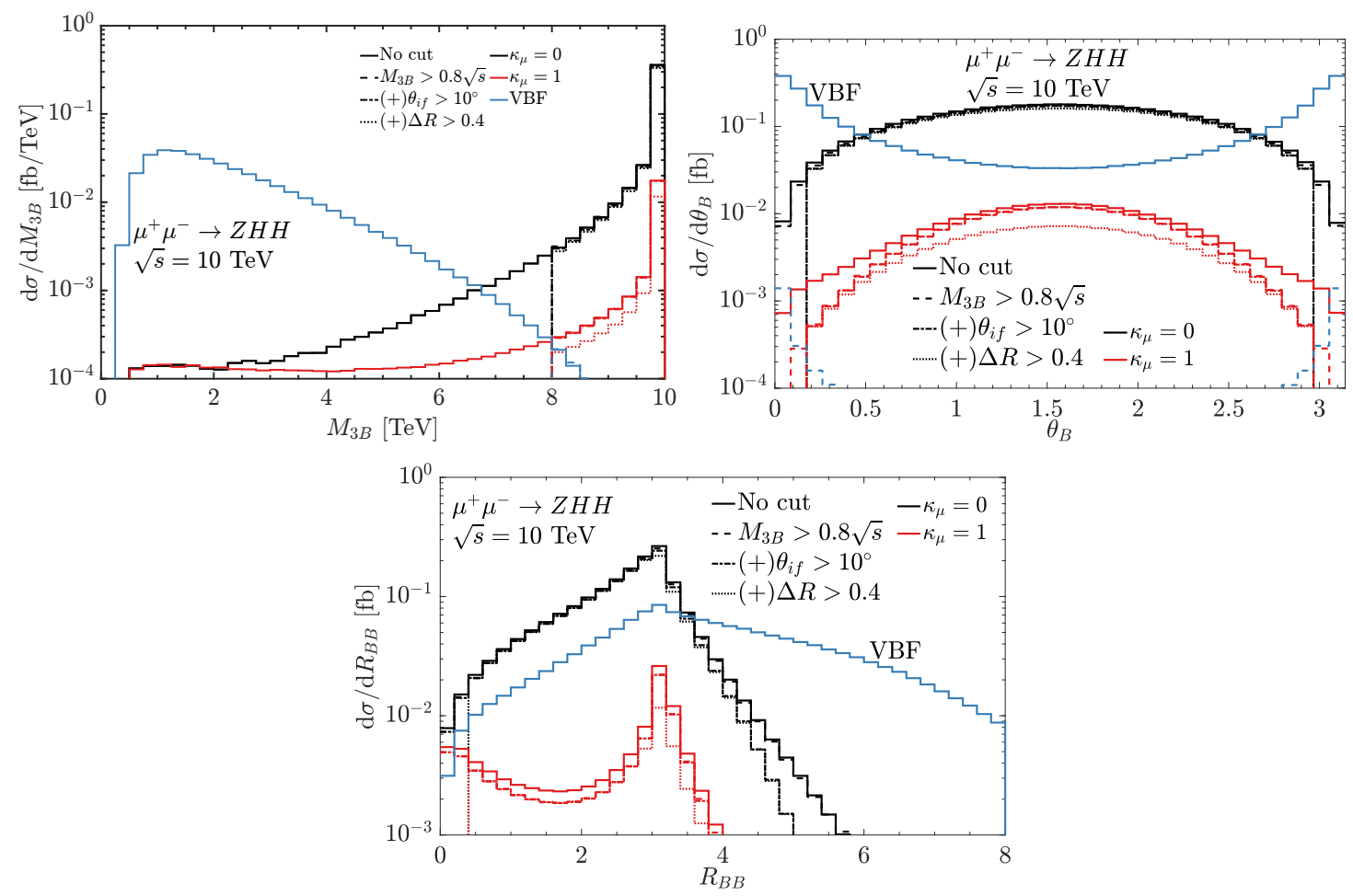

Figure 10. The kinematic distributions for $\theta_{B}, R_{B B}$, and $M_{3 B}$ as in figure 9 , but for $Z H H$ production at a $\sqrt{s}=10 \mathrm{TeV} \mu^{+} \mu^{-}$collider.

plane, and triboson invariant masses $M_{3 B}$, respectively, in figure 9 and 10 . We see two main differences. First, the invariant mass $M_{3 B}$ for the annihilation process is sharply peaked at the collision energy $\sqrt{s}$ seen in figure 9 (a) and 10(a), with a small spread due to the initial-state radiation (ISR). In contrast, in vector-boson fusion, the $M_{3 B}$ is mainly peaked around the threshold. This feature enables us to efficiently separate these two processes and reduce the VBF background with an invariant mass cut. More specifically, with the $M_{3 B}>0.8 \sqrt{s}$ cut, the VBF background is reduced by three orders of magnitudes, with the absolute differential cross sections falling below the lower axis limits in figures 9 and 10 . In comparison, the signal, $\kappa_{\mu}=0(2)$, almost remains the same size, with specific numbers listed in table 4. We also include the cut flow for the cross sections of SM annihilation to $W W H$ and $Z H H$ without including the ISR effect in table 4 . We see the invariant mass cut does not impact at all in this case, because the $M_{3 B}=\sqrt{s}$ is exact as a result of the momentum conservation. Another important observation is that the invariant mass cut $M_{3 B}>0.8 \sqrt{s}$ together with the ISR effect gives roughly the same cross sections without ISR, which justifies neglecting the ISR effect when necessary.

Second, the final-state particles produced in the vector boson fusion are very forward, shown in figure 9 (b) and 10(b). In comparison, the annihilation-produced particles are much more central, especially for the events induced by a Yukawa interaction with $\kappa_{\mu}=$ 0 (2). With an angular cut, such as $10^{\circ}<\theta_{B}<170^{\circ}$ based on the detector design [22], we are able to reduce the VBF background by more than another factor of 10 . The 


\begin{tabular}{|c|c|c|c|c|c|}
\hline Cut flow & $\kappa_{\mu}=1$ & w/o ISR & $\kappa_{\mu}=0(2)$ & CVBF & NVBF \\
\hline$\sigma[\mathrm{fb}]$ & \multicolumn{5}{|c|}{$W W H$} \\
\hline No cut & 0.24 & 0.21 & 0.47 & 2.3 & 7.2 \\
$M_{3 B}>0.8 \sqrt{s}$ & 0.20 & 0.21 & 0.42 & $5.5 \cdot 10^{-3}$ & $3.7 \cdot 10^{-2}$ \\
$10^{\circ}<\theta_{B}<170^{\circ}$ & 0.092 & 0.096 & 0.30 & $2.5 \cdot 10^{-4}$ & $2.7 \cdot 10^{-4}$ \\
$\Delta R_{B B}>0.4$ & 0.074 & 0.077 & 0.28 & $2.1 \cdot 10^{-4}$ & $2.4 \cdot 10^{-4}$ \\
\hline$\#$ of events & 740 & 770 & 2800 & 2.1 & 2.4 \\
\hline$S / B$ & \multicolumn{5}{|c|}{2.8} \\
\hline$\sigma[\mathrm{fb}]$ & $6.9 \cdot 10^{-3}$ & $6.1 \cdot 10^{-3}$ & 0.119 & $9.6 \cdot 10^{-2}$ & $6.7 \cdot 10^{-4}$ \\
\hline No cut & $5.9 \cdot 10^{-3}$ & $6.1 \cdot 10^{-3}$ & 0.115 & $1.5 \cdot 10^{-4}$ & $7.4 \cdot 10^{-6}$ \\
$M_{3 B}>0.8 \sqrt{s}$ & $5.7 \cdot 10^{-3}$ & $6.0 \cdot 10^{-3}$ & 0.110 & $8.8 \cdot 10^{-6}$ & $7.5 \cdot 10^{-7}$ \\
$10^{\circ}<\theta_{B}<170^{\circ}$ & 5.70 & 0.106 & $8.0 \cdot 10^{-6}$ & $5.6 \cdot 10^{-7}$ \\
\hline$R_{B B}>0.4$ & $3.8 \cdot 10^{-3}$ & $4.0 \cdot 10^{-3}$ & 0.060 & - \\
\hline$\#$ of events & 38 & 40 & 1060 & - \\
\hline$S / B$ & \multicolumn{5}{|c|}{} \\
\hline
\end{tabular}

Table 4. The cut-flow for the cross sections of $W W H$ and $Z H H$ production through annihilation (SM with $\kappa_{\mu}=1$ ) with and without ISR, and the BSM signal models for $\kappa_{\mu}=0$ (2) (i.e., $\Delta \kappa_{\mu}= \pm 1$ ). The last two columns are the SM backgrounds from charged (CVBF) and neutral vector boson fusion $(\mathrm{NVBF})$, respectively. All cross sections are at a $\sqrt{s}=10 \mathrm{TeV} \mu^{+} \mu^{-}$collider. The event numbers correspond to an integrated luminosity $\mathcal{L}=10 \mathrm{ab}^{-1}$. The signal and background are defined in eq. (3.6).

SM annihilation cross section will be suppressed by a factor of 2 for $W W H$, while the signal events with $\kappa_{\mu}=0(2)$ are only reduced by $30 \%$. As for the case of the $Z H H$ processes, the impact of the angular cut is small both for the VBF background and for the annihilation process.

Finally, in order to reasonably resolve the final states within the detector, we need to require a basic separation among the reconstructed final-state bosons. The distributions of separation distance $R_{B B}$ in the $W W H$ and $Z H H$ production are shown in figure 9(c) and 10(c). Besides the peak around $R_{B B} \sim \pi$ due to the back-to-back configuration, we obtain another minor peak around $R_{B B} \sim 0$ for the SM annihilations, which reflects the collinear splitting behaviors, such as $W \rightarrow W H$ or $Z \rightarrow Z H$. With a reasonable separation cut $R_{B B}>0.4$, the SM annihilation to $Z H H$ is reduced by roughly $30 \%$ due to the removal of radiation patterns with collinear splitting $Z \rightarrow Z H$. In comparison, both signal and backgrounds for $W W H$ production are only reduced slightly, with specific numbers presented in table 4 . In this case, the collinear splitting coincides with the forward beam region, which is already cut away by the angular acceptance.

\subsection{Statistical sensitivity on the muon Yukawa coupling}

With the integrated luminosity in eq. (3.1), we obtain the event numbers for annihilation and VBF for $W W H$ and $Z H H$, listed in table 4 . We see a big visible deviation from the 

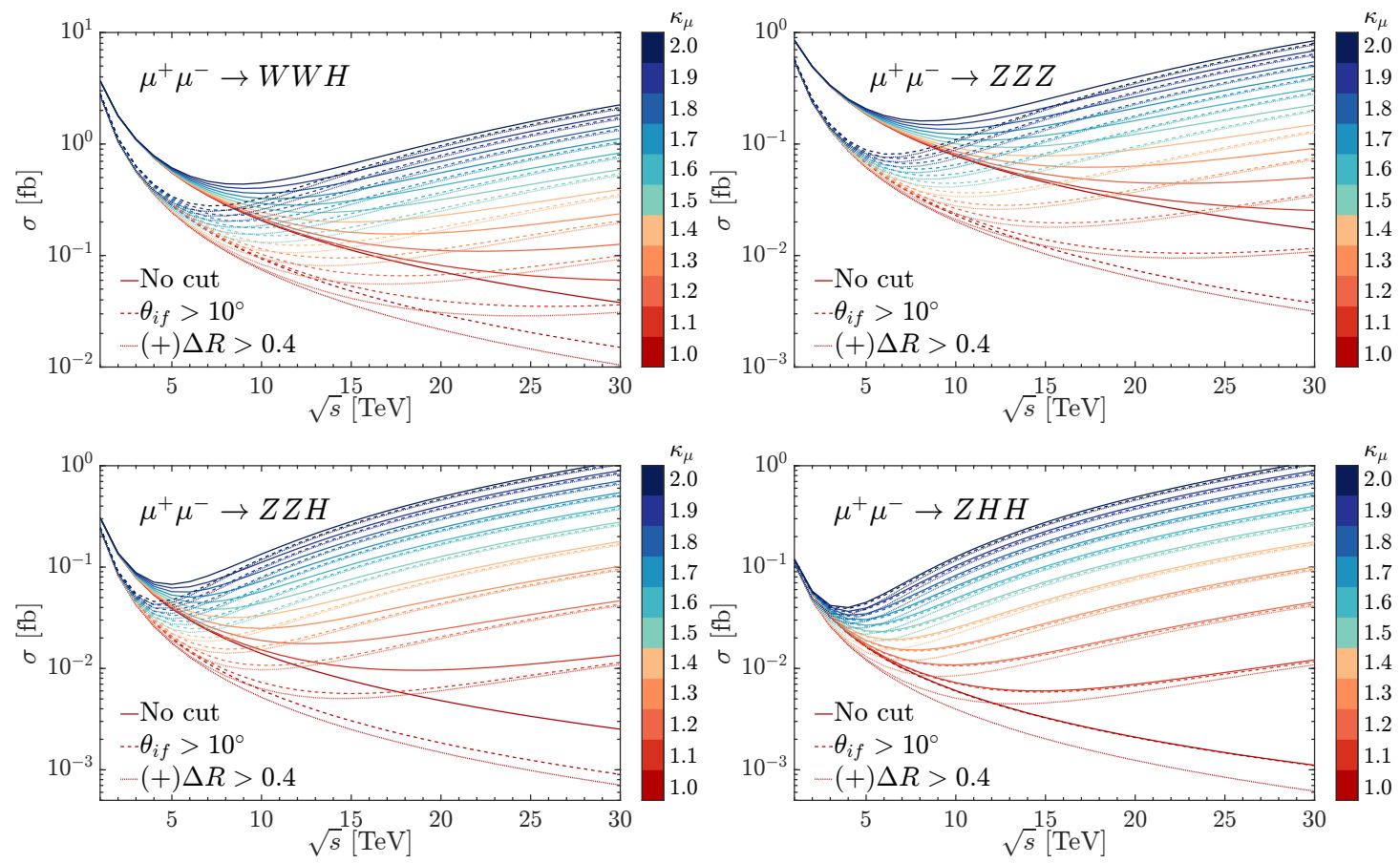

Figure 11. The cross sections of annihilation without ISR for the three-boson production channels $\mu^{+} \mu^{-} \rightarrow W W H, Z Z Z, Z Z H, Z H H$ versus the $\mu^{+} \mu^{-}$c.m. energy $\sqrt{s}$ and the effective coupling $\kappa_{\mu}$. The lower two clusters of curves correspond the flow cut: $\theta_{i f}>10^{\circ}$ and the accumulated $\Delta R>0.4$.

SM backgrounds $\left(\kappa_{\mu}=1\right)$ if we assume the muon Yukawa coupling varying within a range $\kappa_{\mu}=0 \ldots 1 \ldots 2$. We can obtain the signal and background events as

$$
S=N_{\kappa_{\mu}}-N_{\kappa_{\mu}=1}, B=N_{\kappa_{\mu}=1}+N_{\mathrm{VBF}}
$$

with a large signal-to-background ratio $S / B$ for $W W H$ and $Z H H$ shown in table 4 . We can define the corresponding statistical sensitivity to the anomalous (non-SM) muon Yukawa coupling as

$$
\mathcal{S}=\frac{S}{\sqrt{B}}
$$

We would like to emphasize that $\mathcal{S}$ is always positive due to $N_{\kappa_{\mu}} \geq N_{\kappa_{\mu}=1}$, so we can define it without a modulus. We would expect a big sensitivity under the assumption $\kappa_{\mu}=0(2)$ for both $W W H$ and $Z H H$ channels, with the specific values even beyond the applicability of Gaussian approximation adopted in eq. (3.7).

We want to know how precisely we can measure the muon Yukawa coupling at a highenergy muon collider. For this task, we perform a scan of the annihilation cross sections over the collision energy $\sqrt{s}$ and the effective coupling $\kappa_{\mu}$, with results in the band of curves shown in figure 11. We do not include the $W W Z$ channel as the corresponding sensitivity is small resulting from the relatively small deviation shown in figure 5. The ISR effect is safely discarded in this scan, thanks to the balance of the invariant mass cut, illustrated by the example of $W W H$ and $Z H H$ production in table 4 . In figure 11, we present three clusters of curves to illustrate the impact of the cut flow. The solid lines 


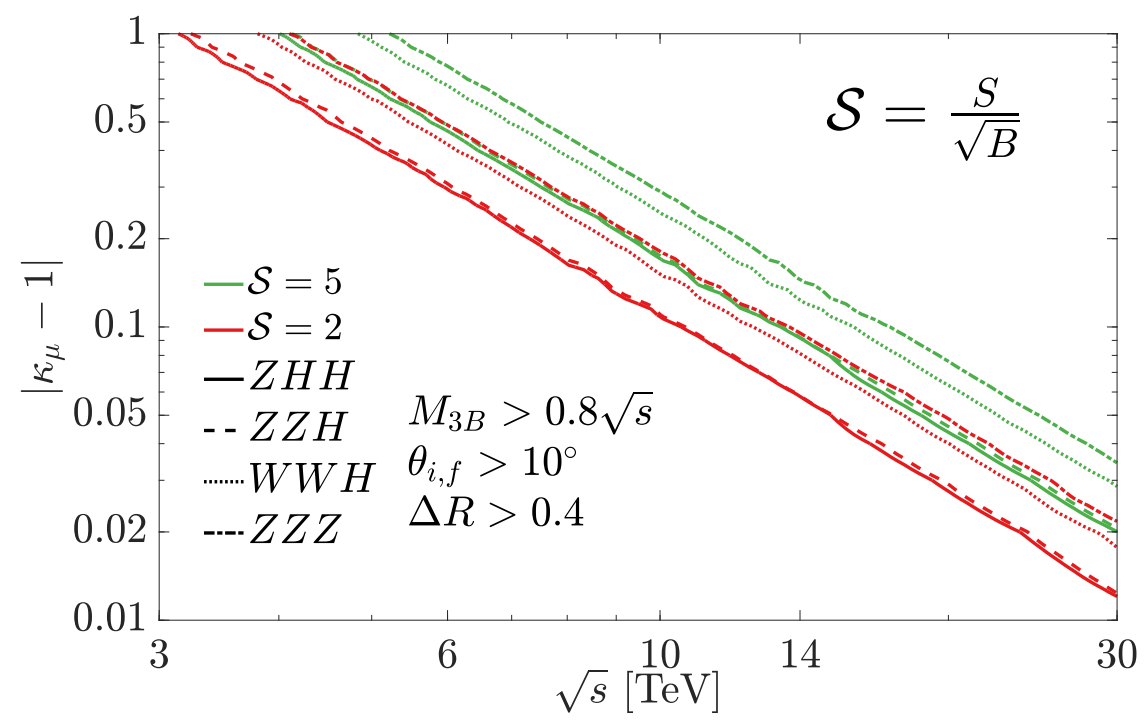

Figure 12. The statistical sensitivity of a high-energy muon collider to the muon Yukawa coupling $\kappa_{\mu}$ from the measurements of three-boson production.

indicate the annihilation cross sections without any cuts. The lower clusters of dashed and dotted curves correspond to the angular cuts $10^{\circ}<\theta_{B}<170^{\circ}$ and the accumulated $\Delta R_{B B}>0.4$. We see that at large collision energy, the signal cross sections corresponding to $\kappa_{\mu} \neq 1$ are not hampered by the kinematic cuts compared to the SM annihilation ones $\left(\kappa_{\mu}=1\right)$. Especially at a large $\kappa_{\mu}$ deviation, such as $\kappa_{\mu}=0(2)$, the cross sections with and without selection cuts are more or less the same. The angular cut almost has no impact on the $Z H H$ channel, because both the $Z$ and $H$ boson are predominantly central in this channel, as mentioned above and shown in figure 10 (b). Instead, the separation distance cut reduces the SM annihilation rate by a factor of $30 \% \sim 40 \%$, due to the removal of collinear splittings of $Z \rightarrow Z H$.

At this stage, we are able to obtain the sensitivity of a high-energy muon collider on the muon Yukawa coupling, by combining the cross sections with the corresponding integrated luminosity. In figure 12, we show two type of contours, corresponding to $\mathcal{S}=2$ and 5 respectively, with an integrated luminosity as given in eq. (3.1). We recall that the sensitivity respects a symmetry that $\left.\mathcal{S}\right|_{\kappa_{\mu}=1+\delta}=\left.\mathcal{S}\right|_{\kappa_{\mu}=1-\delta}$, due to the nature of the symmetric cross sections in eq. (3.5). The channels - in decreasing size of sensitivity - are $Z H H, Z Z H, W W H$, and $Z Z Z$, respectively. At the low energy end, around $3 \mathrm{TeV}$, we are able to probe the muon Yukawa coupling about $100 \%$ by means of the $Z H H$ channel, if we take the criterion $\mathcal{S}=2$. At a 10 (30) $\mathrm{TeV}$ muon collider, we are able to test the muon Yukawa coupling to a precision of up to $10 \%$ (1\%), mostly because of two factors: large signal-to-background ratios and large integrated luminosity. In addition, we see the sensitivity of the $Z Z H$ is very close to the $Z H H$ channel, as a result of the Goldstone equivalence theorem. Again, in the SMEFT formalism, the anticipated precision of $10 \%-1 \%$ would translate to the sensitivity of the scale as $\Lambda \sim 30-100 \mathrm{TeV}$.

So far in this paper, we have focused on the sensitivity to the muon Yukawa coupling 
from triboson production measurements at a high-energy muon collider. Similar analyses can be performed in the two- and four-boson channels. However, the sensitivities from the two-boson channels are expected to be weaker, due to the relatively smaller sizes of the cross-section deviations from anomalous couplings, shown in figure 4 . Though in the fourboson channels, the signal-to-background ratios can be larger than that for the triboson channels, the production rates become significantly smaller compared to the three-boson channels. This elevates in our opinion the triple production to the "golden channels" for this kind of measurement. Our event selection is based on imposing an invariant mass cut $M_{3 B}>0.8 \sqrt{s}$ in our analysis to enrich the annihilation channels. An opposite selection cut could likewise yield enriched samples of VBF processes; this is also expected to have some sensitivity on anomalous muon-Higgs couplings, based on the deviations shown in figure 7(b). As a final remark, annihilation cross sections of (pure) multi-Higgs production do not respect the symmetry in eq. (3.5), which provides an opportunity to determine the sign of the deviation $\delta=\kappa_{\mu}-1$. Nevertheless, the production rate is so small that not even a single expected event survives the event selection, given the luminosity in eq. (3.1). The only chance lies in the single Higgs production with collision energy right on the Higgs mass threshold. We leave all these possibilities to future dedicated studies.

To summarize our results, a high-energy muon collider in the range of $10-30 \mathrm{TeV}$, combining multi-TeV resolution power with the well-defined and clean leptonic environment, allows probing a tiny and elusive parameter of the SM like the muon Yukaww coupling to the single-digit percent level.

\section{Summary and conclusions}

Motivated by the recent proposal for a multi-TeV muon collider, we explored the sensitivity of testing the muon-Higgs coupling at such a collider. Owing to the small muon-Yukawa coupling in the SM, any new physics contributions to the muon mass generation different from the SM Yukawa formalism would result in relatively large deviations from the SM prediction, and thus deserve special scrutiny at future collider experiments. We claim that a muon collider would be unique in carrying out such explorations. Our results are summarized as follows.

After presenting the scale-dependence of the muon Yukawa coupling in the SM and in an extra-dimensional theory, we discussed parameterizations for deviations of the muonYukawa coupling from its SM values within the frameworks of HEFT and SMEFT effective descriptions, and considered the implications on such anomalous couplings from perturbative unitarity bounds. As paradigm observables, we applied this EFT formalism to multi-boson production at a muon collider, particularly the production of two, three and four electroweak gauge bosons associated with a Higgs boson. Using the Goldstone boson equivalence theorem, we derived the scaling behavior of cross sections for processes with multiple bosons, containing deviations to the muon-Higgs coupling, normalized to specific reference cross sections for each multiplicity in section 2.2.4. Our studies show that the sensitivity reach to such anomalous muon-Higgs couplings rises with the number of gauge bosons as the onset of the deviation from the SM is at lower energies. This is due to the fact 
that processes with higher multiplicities are involved in more insertions of the operators generating the deviations (and of higher operators) with high-energy enhancements and sizeable coupling coefficients.

With the approach of a model independent effective coupling $\kappa_{\mu}$, we further performed detailed numerical analyses in section 3, and found that two-boson production processes have less sensitivity to the muon-Yukawa coupling, while those for four-boson production have lower production rates. Therefore, to demonstrate the feasibility of such a study, we identified the optimal processes of triboson production $\mu^{+} \mu^{-} \rightarrow W^{+} W^{-} H, Z H H$ as prime examples and showed how to isolate this from its most severe background, the same final state produced in vector-boson fusion. Typical observables are diboson correlations, either their invariant masses, their angular distributions or their $\Delta R$ distances. In this scenario, a muon collider with up to $30 \mathrm{TeV}$ center-of-mass energy has a sensitivity to deviations of the muon-Yukawa coupling from its SM value of the order of $1 \% \sim 4 \%$. This can be interpreted in the SM as a measurement of the muon Yukawa coupling with this precision. In the SMEFT formulation, if we assume an order-1 coupling, this precision would correspond to a probe to a new physics scale of about $\Lambda \sim 30-100 \mathrm{TeV}$.

There are many ways such an analysis can be improved, e.g., by combining different channels, performing measurements at different energy stages of the machines, by combining final states with different multiplicities, by using multivariate analyses instead of simple cut-based analyses and by using polarization information on the final-state vector bosons. All of this is beyond the scope of this paper and is left for future investigations.

This paper highlights the tantamount possibilities to study one of the most elusive parameters within particle physics, the Higgs-muon coupling, and it also shows in more general context how effective field theories can be utilized to make the utmost use of a discovery facility like the muon collider.

\section{Acknowledgments}

We thank Fabio Maltoni, Daniel Schulte and Andrea Wulzer for useful discussions. This work was supported in part by the U.S. Department of Energy under grant No. DE-FG0295ER40896, U.S. National Science Foundation under Grant No. PHY-1820760, and in part by the PITT PACC. JRR acknowledges the support by the Deutsche Forschungsgemeinschaft (DFG, German Research Association) under Germany's Excellence Strategy-EXC 2121 "Quantum Universe"-39083330. WK and NK were supported in part by the Deutsche Forschungsgemeinschaft (DFG, German Research Foundation) under grant 396021762 TRR 257.

Open Access. This article is distributed under the terms of the Creative Commons Attribution License (CC-BY 4.0), which permits any use, distribution and reproduction in any medium, provided the original author(s) and source are credited. 


\section{References}

[1] Particle Data Group collaboration, Review of particle physics, PTEP 2020 (2020) 083C01 [INSPIRE].

[2] ATLAS collaboration, Observation of a new particle in the search for the standard model Higgs boson with the ATLAS detector at the LHC, Phys. Lett. B 716 (2012) 1 [arXiv: 1207.7214] [INSPIRE].

[3] CMS collaboration, Observation of a new boson at a mass of $125 \mathrm{GeV}$ with the CMS experiment at the LHC, Phys. Lett. B $\mathbf{7 1 6}$ (2012) 30 [arXiv:1207.7235] [INSPIRE].

[4] LHC Higgs Cross Section Working Group collaboration, Handbook of LHC Higgs cross sections: 4. Deciphering the nature of the Higgs sector, arXiv:1610.07922 [INSPIRE].

[5] J. de Blas et al., Higgs boson studies at future particle colliders, JHEP 01 (2020) 139 [arXiv: 1905. 03764] [INSPIRE].

[6] ATLAS collaboration, Projections for measurements of Higgs boson cross sections, branching ratios, coupling parameters and mass with the ATLAS detector at the HL-LHC, ATL-PHYS-PUB-2018-054 (2018).

[7] CMS collaboration, Sensitivity projections for Higgs boson properties measurements at the HL-LHC, CMS-PAS-FTR-18-011 (2018).

[8] R.K. Ellis et al., Physics briefing book: input for the European strategy for particle physics update 2020, arXiv:1910.11775 [INSPIRE].

[9] European Strategy Group collaboration, 2020 Update of the European Strategy for Particle Physics, CERN Council, Geneva (2020), https://doi.org/10.17181/ESU2020.

[10] H. Baer et al., eds., The International Linear Collider technical design report - Volume 2: physics, arXiv:1306.6352 [INSPIRE].

[11] H. Abramowicz et al., The International Linear Collider technical design report - Volume 4: detectors, arXiv:1306.6329 [INSPIRE].

[12] FCC collaboration, FCC-ee: the lepton collider: Future Circular Collider conceptual design report volume 2, Eur. Phys. J. ST 228 (2019) 261 [INSPIRE].

[13] CEPC Study Group collaboration, CEPC conceptual design report: volume 2 - Physics \& Detector, arXiv:1811.10545 [INSPIRE].

[14] M. Aicheler et al., A multi-TeV linear collider based on CLIC technology: CLIC conceptual design report, CERN-2012-007 (2012).

[15] CLIC, CLICDP collaboration, Updated baseline for a staged Compact Linear Collider, arXiv: 1608.07537 [INSPIRE].

[16] CMS collaboration, Evidence for Higgs boson decay to a pair of muons, JHEP 01 (2021) 148 [arXiv: 2009.04363] [INSPIRE].

[17] ATLAS collaboration, A search for the dimuon decay of the Standard Model Higgs boson with the ATLAS detector, Phys. Lett. B 812 (2021) 135980 [arXiv:2007.07830] [InSPIRE].

[18] ATLAS collaboration, Projections for measurements of Higgs boson signal strengths and coupling parameters with the ATLAS detector at a HL-LHC,ATL-PHYS-PUB-2014-016 (2014).

[19] FCC collaboration, FCC physics opportunities: Future Circular Collider conceptual design report volume 1, Eur. Phys. J. C 79 (2019) 474 [INSPIRE]. 
[20] FCC collaboration, FCC-hh: the hadron collider: Future Circular collider conceptual design report volume 3, Eur. Phys. J. ST 228 (2019) 755 [INSPIRE].

[21] J.P. Delahaye et al., Muon colliders, arXiv:1901.06150 [INSPIRE].

[22] N. Bartosik et al., Detector and physics performance at a muon collider, 2020 JINST 15 P05001 [arXiv:2001.04431] [INSPIRE].

[23] D. Schulte et al., Prospects on Muon Colliders, PoS(ICHEP2020) 703 [INSPIRE].

[24] K.R. Long, D. Lucchesi, M.A. Palmer, N. Pastrone, D. Schulte and V. Shiltsev, Muon colliders to expand frontiers of particle physics, Nature Phys. 17 (2021) 289.

[25] T. Han, Y. Ma and K. Xie, High energy leptonic collisions and electroweak parton distribution functions, Phys. Rev. D 103 (2021) L031301 [arXiv:2007.14300] [INSPIRE].

[26] A. Costantini et al., Vector boson fusion at multi-TeV muon colliders, JHEP 09 (2020) 080 [arXiv:2005.10289] [INSPIRE].

[27] D. Buttazzo, R. Franceschini and A. Wulzer, Two paths towards precision at a very high energy lepton collider, JHEP 05 (2021) 219 [arXiv:2012.11555] [INSPIRE].

[28] T. Han, Y. Ma and K. Xie, Quark and gluon contents of a lepton at high energies, arXiv:2103.09844 [INSPIRE].

[29] D. Buarque et al., Vector boson scattering processes: status and prospects, arXiv:2106.01393 [INSPIRE].

[30] T. Han, D. Liu, I. Low and X. Wang, Electroweak couplings of the Higgs boson at a multi-TeV muon collider, Phys. Rev. D 103 (2021) 013002 [arXiv:2008.12204] [INSPIRE].

[31] T. Han, Z. Liu, L.-T. Wang and X. Wang, WIMPs at high energy muon colliders, Phys. Rev. D 103 (2021) 075004 [arXiv:2009.11287] [INSPIRE].

[32] T. Han, S. Li, S. Su, W. Su and Y. Wu, Heavy Higgs bosons in 2HDM at a muon collider, Phys. Rev. D 104 (2021) 055029 [arXiv:2102.08386] [INSPIRE].

[33] R. Capdevilla, D. Curtin, Y. Kahn and G. Krnjaic, Discovering the physics of $(g-2)_{\mu}$ at future muon colliders, Phys. Rev. D 103 (2021) 075028 [arXiv:2006.16277] [InSPIRE].

[34] W. Yin and M. Yamaguchi, Muon g-2 at multi-TeV muon collider, arXiv:2012.03928 [INSPIRE].

[35] R. Capdevilla, D. Curtin, Y. Kahn and G. Krnjaic, A No-Lose Theorem for Discovering the New Physics of $(g-2)_{\mu}$ at Muon Colliders, arXiv:2101.10334 [INSPIRE].

[36] W. Liu and K.-P. Xie, Probing electroweak phase transition with multi-TeV muon colliders and gravitational waves, JHEP 04 (2021) 015 [arXiv:2101.10469] [INSPIRE].

[37] J. Gu, L.-T. Wang and C. Zhang, An unambiguous test of positivity at lepton colliders, arXiv:2011.03055 [INSPIRE].

[38] G.-y. Huang, F.S. Queiroz and W. Rodejohann, Gauged $L_{\mu}-L_{\tau}$ at a muon collider, Phys. Rev. D 103 (2021) 095005 [arXiv:2101.04956] [INSPIRE].

[39] R. Capdevilla, F. Meloni, R. Simoniello and J. Zurita, Hunting wino and higgsino dark matter at the muon collider with disappearing tracks, JHEP 06 (2021) 133 [arXiv:2102.11292] [INSPIRE].

[40] MuON G-2 collaboration, Final report of the Muon E821 anomalous magnetic moment measurement at BNL, Phys. Rev. D 73 (2006) 072003 [hep-ex/0602035] [InSPIRE]. 
[41] Muon G-2 collaboration, Measurement of the positive muon anomalous magnetic moment to 0.46 ppm, Phys. Rev. Lett. 126 (2021) 141801 [arXiv:2104.03281] [INSPIRE].

[42] M.E. Machacek and M.T. Vaughn, Two loop renormalization group equations in a general quantum field theory. 1. Wave function renormalization, Nucl. Phys. B 222 (1983) 83 [INSPIRE].

[43] M.E. Machacek and M.T. Vaughn, Two loop renormalization group equations in a general quantum field theory. 2. Yukawa couplings, Nucl. Phys. B 236 (1984) 221 [inSPIRE].

[44] H. Arason et al., Top quark and Higgs mass bounds from a numerical study of superGUTs, Phys. Rev. Lett. 67 (1991) 2933 [INSPIRE].

[45] H. Arason et al., Renormalization group study of the standard model and its extensions. 1. The Standard model, Phys. Rev. D 46 (1992) 3945 [INSPIRE].

[46] H. Arason, D.J. Castano, E.J. Piard and P. Ramond, Mass and mixing angle patterns in the standard model and its minimal supersymmetric extension, Phys. Rev. D 47 (1993) 232 [hep-ph/9204225] [INSPIRE].

[47] D.J. Castano, E.J. Piard and P. Ramond, Renormalization group study of the Standard Model and its extensions. 2. The minimal supersymmetric standard model, Phys. Rev. D 49 (1994) 4882 [hep-ph/9308335] [INSPIRE].

[48] B. Grzadkowski and M. Lindner, Nonlinear evolution of Yukawa couplings, Phys. Lett. B 193 (1987) 71 [INSPIRE].

[49] W. Altmannshofer, S. Gori, A.L. Kagan, L. Silvestrini and J. Zupan, Uncovering mass generation through Higgs flavor violation, Phys. Rev. D 93 (2016) 031301 [arXiv: 1507.07927] [INSPIRE].

[50] Y. Soreq, H.X. Zhu and J. Zupan, Light quark Yukawa couplings from Higgs kinematics, JHEP 12 (2016) 045 [arXiv: 1606.09621] [INSPIRE].

[51] G.T. Bodwin, F. Petriello, S. Stoynev and M. Velasco, Higgs boson decays to quarkonia and

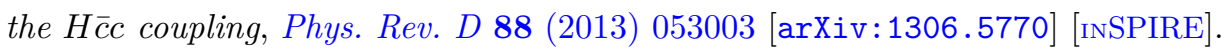

[52] A.L. Kagan, G. Perez, F. Petriello, Y. Soreq, S. Stoynev and J. Zupan, Exclusive window onto Higgs Yukawa couplings, Phys. Rev. Lett. 114 (2015) 101802 [arXiv:1406.1722] [INSPIRE].

[53] G. Perez, Y. Soreq, E. Stamou and K. Tobioka, Constraining the charm Yukawa and Higgs-quark coupling universality, Phys. Rev. D 92 (2015) 033016 [arXiv:1503.00290] [INSPIRE].

[54] F. Bishara, U. Haisch, P.F. Monni and E. Re, Constraining light-quark Yukawa couplings from Higgs distributions, Phys. Rev. Lett. 118 (2017) 121801 [arXiv:1606.09253] [INSPIRE].

[55] J. Duarte-Campderros, G. Perez, M. Schlaffer and A. Soffer, Probing the Higgs-strange-quark coupling at $e^{+} e^{-}$colliders using light-jet flavor tagging, Phys. Rev. D 101 (2020) 115005 [arXiv: 1811.09636] [INSPIRE].

[56] W. Altmannshofer, J. Brod and M. Schmaltz, Experimental constraints on the coupling of the Higgs boson to electrons, JHEP 05 (2015) 125 [arXiv: 1503.04830] [INSPIRE].

[57] R. Harnik, J. Kopp and J. Zupan, Flavor violating Higgs decays, JHEP 03 (2013) 026 [arXiv: 1209.1397] [INSPIRE]. 
[58] K.R. Dienes, E. Dudas and T. Gherghetta, Extra space-time dimensions and unification, Phys. Lett. B 436 (1998) 55 [hep-ph/9803466] [INSPIRE].

[59] K.R. Dienes, E. Dudas and T. Gherghetta, Grand unification at intermediate mass scales through extra dimensions, Nucl. Phys. B 537 (1999) 47 [hep-ph/9806292] [INSPIRE].

[60] T. Appelquist, H.-C. Cheng and B.A. Dobrescu, Bounds on universal extra dimensions, Phys. Rev. D 64 (2001) 035002 [hep-ph/0012100] [InSPIRE].

[61] T. Appelquist, B.A. Dobrescu, E. Ponton and H.-U. Yee, Proton stability in six-dimensions, Phys. Rev. Lett. 87 (2001) 181802 [hep-ph/0107056] [INSPIRE].

[62] G. Bhattacharyya, A. Datta, S.K. Majee and A. Raychaudhuri, Power law blitzkrieg in universal extra dimension scenarios, Nucl. Phys. B $\mathbf{7 6 0}$ (2007) 117 [hep-ph/0608208] [INSPIRE].

[63] A.S. Cornell, A. Deandrea, L.-X. Liu and A. Tarhini, Renormalisation running of masses and mixings in UED models, Mod. Phys. Lett. A 28 (2013) 1330007 [arXiv:1209.6239] [INSPIRE].

[64] M. Blennow, H. Melbeus, T. Ohlsson and H. Zhang, Renormalization Group Running of the Neutrino Mass Operator in Extra Dimensions, JHEP 04 (2011) 052 [arXiv:1101.2585] [INSPIRE].

[65] T. Kakuda, K. Nishiwaki, K.-y. Oda and R. Watanabe, Universal extra dimensions after Higgs discovery, Phys. Rev. D 88 (2013) 035007 [arXiv:1305.1686] [InSPIRE].

[66] A. Abdalgabar, A.S. Cornell, A. Deandrea and A. Tarhini, Evolution of Yukawa couplings and quark flavour mixings in 2UED models, Phys. Rev. D 88 (2013) 056006 [arXiv: 1306 .4852] [inSPIRE].

[67] A.S. Cornell, A. Deandrea, L.-X. Liu and A. Tarhini, Scaling of the CKM Matrix in the 5D MSSM, Phys. Rev. D 85 (2012) 056001 [arXiv:1110.1942] [INSPIRE].

[68] S.R. Coleman, J. Wess and B. Zumino, Structure of phenomenological Lagrangians. 1, Phys. Rev. 177 (1969) 2239 [INSPIRE].

[69] C.G. Callan, Jr., S.R. Coleman, J. Wess and B. Zumino, Structure of phenomenological Lagrangians. 2, Phys. Rev. 177 (1969) 2247 [INSPIRE].

[70] S. Weinberg, Effective gauge theories, Phys. Lett. B 91 (1980) 51 [InSPIRE].

[71] T. Appelquist and C.W. Bernard, Strongly interacting Higgs bosons, Phys. Rev. D 22 (1980) 200 [INSPIRE].

[72] A.C. Longhitano, Low-energy impact of a heavy Higgs boson sector, Nucl. Phys. B 188 (1981) 118 [INSPIRE].

[73] A. Dobado, D. Espriu and M.J. Herrero, Chiral Lagrangians as a tool to probe the symmetry breaking sector of the SM at LEP, Phys. Lett. B 255 (1991) 405 [INSPIRE].

[74] M.S. Chanowitz and M.K. Gaillard, The TeV physics of strongly interacting W's and Z's, Nucl. Phys. B 261 (1985) 379 [INSPIRE].

[75] G.J. Gounaris, R. Kogerler and H. Neufeld, Relationship between longitudinally polarized vector bosons and their unphysical scalar partners, Phys. Rev. D 34 (1986) 3257 [InSPIRE].

[76] A. Dobado, A. Gomez-Nicola, A.L. Maroto and J.R. Pelaez, Effective lagrangians for the standard model, Texts and Monographs in Physics, Springer, Germany (1997). 
[77] A. Manohar and H. Georgi, Chiral quarks and the nonrelativistic quark model, Nucl. Phys. B 234 (1984) 189 [INSPIRE].

[78] A.G. Cohen, D.B. Kaplan and A.E. Nelson, Counting 4 pis in strongly coupled supersymmetry, Phys. Lett. B 412 (1997) 301 [hep-ph/9706275] [INSPIRE].

[79] S. Weinberg, Baryon and lepton nonconserving processes, Phys. Rev. Lett. 43 (1979) 1566 [INSPIRE].

[80] L.F. Abbott and M.B. Wise, The effective Hamiltonian for nucleon decay, Phys. Rev. D 22 (1980) 2208 [INSPIRE].

[81] W. Buchmüller and D. Wyler, Effective Lagrangian analysis of new interactions and flavor conservation, Nucl. Phys. B 268 (1986) 621 [InSPIRE].

[82] B. Grzadkowski, M. Iskrzynski, M. Misiak and J. Rosiek, Dimension-six terms in the Standard Model Lagrangian, JHEP 10 (2010) 085 [arXiv:1008.4884] [INSPIRE].

[83] A. Falkowski et al., Light quark Yukawas in triboson final states, JHEP 04 (2021) 023 [arXiv: 2011.09551] [INSPIRE].

[84] F. Maltoni, J.M. Niczyporuk and S. Willenbrock, The scale of fermion mass generation, Phys. Rev. D 65 (2002) 033004 [hep-ph/0106281] [INSPIRE].

[85] V.D. Barger, M.S. Berger, J.F. Gunion and T. Han, s channel Higgs boson production at a muon muon collider, Phys. Rev. Lett. 75 (1995) 1462 [hep-ph/9504330] [INSPIRE].

[86] V.D. Barger, M.S. Berger, J.F. Gunion and T. Han, Higgs boson physics in the s channel at $\mu^{+} \mu^{-}$colliders, Phys. Rept. 286 (1997) 1 [hep-ph/9602415] [INSPIRE].

[87] N. Chakrabarty, T. Han, Z. Liu and B. Mukhopadhyaya, Radiative return for heavy Higgs boson at a muon collider, Phys. Rev. D 91 (2015) 015008 [arXiv: 1408.5912] [INSPIRE].

[88] R. Kleiss, W.J. Stirling and S.D. Ellis, A new Monte Carlo treatment of multiparticle phase space at high-energies, Comput. Phys. Commun. 40 (1986) 359 [INSPIRE].

[89] W. Kilian, T. Ohl and J. Reuter, WHIZARD: simulating multi-particle processes at LHC and ILC, Eur. Phys. J. C $\mathbf{7 1}$ (2011) 1742 [arXiv:0708.4233] [INSPIRE].

[90] M. Moretti, T. Ohl and J. Reuter, O'Mega: an Optimizing Matrix Element Generator, hep-ph/0102195 [INSPIRE].

[91] S. Brass, W. Kilian and J. Reuter, Parallel adaptive Monte Carlo integration with the event generator WHIZARD, Eur. Phys. J. C 79 (2019) 344 [arXiv:1811.09711] [InSPIRE].

[92] N.D. Christensen, C. Duhr, B. Fuks, J. Reuter and C. Speckner, Introducing an interface between WHIZARD and FeynRules, Eur. Phys. J. C 72 (2012) 1990 [arXiv:1010.3251] [INSPIRE].

[93] A. Alboteanu, W. Kilian and J. Reuter, Resonances and unitarity in weak boson scattering at the LHC, JHEP 11 (2008) 010 [arXiv:0806 .4145] [INSPIRE].

[94] W. Kilian, T. Ohl, J. Reuter and M. Sekulla, High-energy vector boson scattering after the Higgs discovery, Phys. Rev. D 91 (2015) 096007 [arXiv:1408.6207] [InSPIRE].

[95] S. Brass, C. Fleper, W. Kilian, J. Reuter and M. Sekulla, Transversal modes and Higgs bosons in electroweak vector-boson scattering at the LHC, Eur. Phys. J. C 78 (2018) 931 [arXiv: 1807.02512] [INSPIRE].

[96] A. Ballestrero et al., Precise predictions for same-sign W-boson scattering at the LHC, Eur. Phys. J. C 78 (2018) 671 [arXiv:1803.07943] [inSPIRE]. 
[97] M. Beyer et al., Determination of new electroweak parameters at the ILC - Sensitivity to new physics, Eur. Phys. J. C 48 (2006) 353 [hep-ph/0604048] [INSPIRE].

[98] C. Fleper, W. Kilian, J. Reuter and M. Sekulla, Scattering of $W$ and $Z$ bosons at high-energy lepton colliders, Eur. Phys. J. C 77 (2017) 120 [arXiv:1607.03030] [InSPIRE].

[99] E. Boos, H.J. He, W. Kilian, A. Pukhov, C.P. Yuan and P.M. Zerwas, Strongly interacting vector bosons at $\mathrm{TeV} e^{+} e^{-}$linear colliders, Phys. Rev. D 57 (1998) 1553 [hep-ph/9708310] [INSPIRE].

[100] E. Boos, H.J. He, W. Kilian, A. Pukhov, C.P. Yuan and P.M. Zerwas, Strongly interacting vector bosons at TeV $e^{ \pm} e^{-}$linear colliders: Addendum, Phys. Rev. D 61 (2000) 077901 [hep-ph/9908409] [INSPIRE]. 